\title{
Amplitude analysis and branching-fraction measurement of $D_{S}^{+} \rightarrow K_{S}^{0} K^{-} \pi^{+} \pi^{+}$
}

M. Ablikim, ${ }^{1}$ M. N. Achasov, ${ }^{10, c}$ P. Adlarson,${ }^{67}$ S. Ahmed,${ }^{15}$ M. Albrecht, ${ }^{4}$ R. Aliberti, ${ }^{28}$ A. Amoroso, ${ }^{66 a, 66 c}$ M. R. An,${ }^{32}$ Q. An ${ }^{63,49}$ X. H. Bai ${ }^{57}$ Y. Bai, ${ }^{48}$ O. Bakina, ${ }^{29}$ R. Baldini Ferroli ${ }^{23 a}$ I. Balossino, ${ }^{24 a}$ Y. Ban,${ }^{38, k}$ K. Begzsuren, ${ }^{26}$ N. Berger, ${ }^{28}$ M. Bertani, ${ }^{23 a}$ D. Bettoni, ${ }^{24 a}$ F. Bianchi ${ }^{66 a, 66 c}$ J. Bloms, ${ }^{60}$ A. Bortone, ${ }^{66 a, 66 c}$ I. Boyko, ${ }^{29}$ R. A. Briere, ${ }^{5}$ H. Cai ${ }^{68}$ X. Cai ${ }^{1,49}$ A. Calcaterra, ${ }^{23 a}$ G. F. Cao, ${ }^{1,54}$ N. Cao, ${ }^{1,54}$ S. A. Cetin, ${ }^{53 a}$ J. F. Chang,,${ }^{1,49}$ W. L. Chang, ${ }^{1,54}$ G. Chelkov, ${ }^{29, b}$ D. Y. Chen $\odot,{ }^{6}$ G. Chen, ${ }^{1}$ H. S. Chen, ${ }^{1,54}$ M. L. Chen, ${ }^{1,49}$ S. J. Chen ${ }^{35}$ X. R. Chen, ${ }^{25}$ Y. B. Chen, ${ }^{1,49}$ Z. J. Chen, ${ }^{20,1}$ W. S. Cheng, ${ }^{60}$ G. Cibinetto, ${ }^{24 a}$ F. Cossio, ${ }^{66 c}$ X. F. Cui ${ }^{36}$ H. L. Dai, ${ }^{1,49}$ X. C. Dai, ${ }^{1,54}$ A. Dbeyssi, ${ }^{15}$ R. E. de Boer, ${ }^{4}$ D. Dedovich ${ }^{29}$ Z. Y. Deng, ${ }^{1}$ A. Denig, ${ }^{28}$ I. Denysenko, ${ }^{29}$ M. Destefanis, ${ }^{66 a, 66 c}$ F. De Mori, ${ }^{66 a, 66 c}$ Y. Ding ${ }^{33}$ C. Dong ${ }^{36}$ J. Dong, ${ }^{1,49}$ L. Y. Dong ${ }^{1,54}$ M. Y. Dong, ${ }^{1,49,54}$ X. Dong, ${ }^{68}$ S. X. Du ${ }^{71}$ Y. L. Fan ${ }^{68}$ J. Fang,${ }^{1,49}$ S. S. Fang, ${ }^{1,54}$ Y. Fang, ${ }^{1}$ R. Farinelli, ${ }^{24 a}$ L. Fava, ${ }^{66 b, 66 c}$ F. Feldbauer, ${ }^{4}$ G. Felici, ${ }^{23 a}$ C. Q. Feng, ${ }^{63,49}$ J. H. Feng, ${ }^{50}$ M. Fritsch, ${ }^{4}$ C. D. Fu, ${ }^{1}$ Y. Gao,${ }^{64}$ Y. Gao,${ }^{63,49}$ Y. Gao, ${ }^{38, k}$ Y. G. Gao,${ }^{6}$ I. Garzia,${ }^{24 a, 24 b}$ P. T. Ge ${ }^{68}$ C. Geng, ${ }^{50}$ E. M. Gersabeck ${ }^{58}$ A. Gilman, ${ }^{61}$ K. Goetzen ${ }^{11}$ L. Gong, ${ }^{33}$ W. X. Gong, ${ }^{1,49}$ W. Gradl, ${ }^{28}$ M. Greco, ${ }^{66,66 c}$ L. M. Gu, ${ }^{35}$ M. H. Gu, ${ }^{1,49}$ S. Gu, ${ }^{2}$ Y. T. Gu, ${ }^{13}$ C. Y. Guan,,${ }^{1,54}$ A. Q. Guo, ${ }^{22}$ L. B. Guo ${ }^{34}$ R. P. Guo, ${ }^{40}$ Y. P. Guo, ${ }^{9, h}$ A. Guskov, ${ }^{29}$ T. T. Han, ${ }^{41}$ W. Y. Han, ${ }^{32}$ X. Q. Hao, ${ }^{16}$ F. A. Harris, ${ }^{56}$ N. Hüsken,${ }^{22,28}$ K. L. He ${ }^{1,54}$ F. H. Heinsius, ${ }^{4}$ C. H. Heinz, ${ }^{28}$ T. Held, ${ }^{4}$ Y. K. Heng, ${ }^{1,49,54}$ C. Herold, ${ }^{51}$ M. Himmelreich, ${ }^{11, f}$ T. Holtmann, ${ }^{4}$ Y. R. Hou,${ }^{54}$ Z. L. Hou, ${ }^{1}$ H. M. Hu, ${ }^{1,54}$ J. F. Hu, ${ }^{47, \mathrm{~m}}$ T. Hu,${ }^{1,49,54}$ Y. Hu, ${ }^{1}$ G. S. Huang, ${ }^{63,49}$ L. Q. Huang, ${ }^{64}$ X. T. Huang ${ }^{41}$ Y. P. Huang, ${ }^{1}$ Z. Huang, ${ }^{38, k}$ T. Hussain, ${ }^{65}$ W. Ikegami Andersson, ${ }^{67}$ W. Imoehl, ${ }^{22}$ M. Irshad, ${ }^{63,49}$ S. Jaeger, ${ }^{4}$ S. Janchiv, ${ }^{26, j}$ Q. Ji, ${ }^{1}$ Q. P. Ji ${ }^{16}$ X. B. Ji, ${ }^{1,54}$ X. L. Ji, ${ }^{1,49}$ H. B. Jiang, ${ }^{41}$ X. S. Jiang, ${ }^{1,49,54}$ J. B. Jiao, ${ }^{41}$ Z. Jiao, ${ }^{18}$ S. Jin,${ }^{35}$ Y. Jin,${ }^{57}$

T. Johansson, ${ }^{67}$ N. Kalantar-Nayestanaki,${ }^{55}$ X. S. Kang, ${ }^{33}$ R. Kappert, ${ }^{55}$ M. Kavatsyuk, ${ }^{55}$ B. C. Ke, ${ }^{43,1}$ I. K. Keshk, ${ }^{4}$ A. Khoukaz,${ }^{60}$ P. Kiese, ${ }^{28}$ R. Kiuchi, ${ }^{1}$ R. Kliemt,${ }^{11}$ L. Koch, ${ }^{30}$ O. B. Kolcu, ${ }^{53 a, e}$ B. Kopf,${ }^{4}$ M. Kuemmel, ${ }^{4}$ M. Kuessner, ${ }^{4}$ A. Kupsc, ${ }^{67}$ M. G. Kurth, ${ }^{1,54}$ W. Kühn, ${ }^{30}$ J. J. Lane, ${ }^{58}$ J. S. Lange ${ }^{30}$ P. Larin ${ }^{15}$ A. Lavania, ${ }^{21}$ L. Lavezzi, ${ }^{66 a, 66 c}$ Z. H. Lei, ${ }^{63,49}$ H. Leithoff, ${ }^{28}$ M. Lellmann, ${ }^{28}$ T. Lenz, ${ }^{28}$ C. Li ${ }^{39}$ C. H. Li ${ }^{32}$ Cheng Li ${ }^{63,49}$ D. M. Li, ${ }^{71}$ F. Li, ${ }^{1,49} \mathrm{G} . \mathrm{Li},{ }^{1}{ }^{1} \mathrm{H} . \mathrm{Li},{ }^{43} \mathrm{H} . \mathrm{Li},{ }^{63,49}$ H. B. Li, ${ }^{1,54}$ H. J. Li, ${ }^{9, h}$ J. L. Li, ${ }^{41}$ J. Q. Li, ${ }^{4}$ J. S. Li, ${ }^{50}$ Ke Li, ${ }^{1}$ L. K. Li,${ }^{1}$ Lei Li ${ }^{3}$ P. R. Li, ${ }^{31}$ S. Y. Li, ${ }^{52}$ W. D. Li,${ }^{1,54}$ W. G. Li ${ }^{1}$ X. H. Li, ${ }^{63,49}$ X. L. Li ${ }^{41}$ Z. Y. Li ${ }^{50}$ H. Liang, ${ }^{63,49}$ H. Liang,,${ }^{1,54}$ H. Liang, ${ }^{27}$ Y. F. Liang, ${ }^{45}$ Y. T. Liang, ${ }^{25}$ L. Z. Liao, ${ }^{1,54}$ J. Libby, ${ }^{21}$ C. X. Lin, ${ }^{50}$ B. J. Liu, ${ }^{1}$ C. X. Liu, ${ }^{1}$ D. Liu,${ }^{63,49}$ F. H. Liu, ${ }^{44}$ Fang Liu, ${ }^{1}$ Feng Liu, ${ }^{6}$ H. B. Liu, ${ }^{13}$ H. M. Liu, ${ }^{1,54}$ Huanhuan Liu, ${ }_{1}^{1}$ Huihui Liu, ${ }^{17}$ J. B. Liu, ${ }^{63,49}$ J. L. Liu, ${ }^{64}$ J. Y. Liu, ${ }^{1,54}$ K. Liu, ${ }^{1}$ K. Y. Liu, ${ }^{33}$ Ke Liu, ${ }^{6}$ L. Liu, ${ }^{63,49}$ M. H. Liu, ${ }^{9}$, P. L. Liu, ${ }^{1}$ Q. Liu ${ }^{54}$ Q. Liu, ${ }^{68}$ S. B. Liu ${ }^{63,49}$ Shuai Liu, ${ }^{46}$ T. Liu, ${ }^{1,54}$ W. M. Liu, ${ }^{63,49}$ X. Liu, ${ }^{31}$ Y. Liu, ${ }^{31}$ Y. B. Liu, ${ }^{36}$ Z. A. Liu,,${ }^{1,49,54}$ Z. Q. Liu, ${ }^{41}$ X. C. Lou,${ }^{1,49,54}$ F. X. Lu, ${ }^{50}$ F. X. Lu, ${ }^{16}$ H. J. Lu, ${ }^{18}$ J. D. Lu, ${ }^{1,54}$ J. G. Lu, ${ }^{1,49}$ X. L. Lu, ${ }^{1}$ Y. Lu, ${ }^{1}$ Y. P. Lu ${ }^{1,49}$ C. L. Luo, ${ }^{34}$ M. X. Luo,${ }^{70}$ P. W. Luo, ${ }^{50}$ T. Luo, ${ }^{9,}$ X. L. Luo, ${ }^{1,49}$ S. Lusso, ${ }^{66 c}$ X. R. Lyu,${ }^{54}$ F. C. Ma, ${ }^{33}$ H. L. Ma, ${ }^{1}$ L. L. Ma ${ }^{41}$ M. M. Ma, ${ }^{1,54}$ Q. M. Ma, ${ }^{1}$ R. Q. Ma, ${ }^{1,54}$ R. T. Ma, ${ }^{54}$ X. X. Ma ${ }^{1,54}$ X. Y. Ma, ${ }^{1,49}$ F. E. Maas, ${ }^{15}$ M. Maggiora, ${ }^{66 a, 66 c}$ S. Maldaner, ${ }^{4}$ S. Malde, ${ }^{61}$ A. Mangoni, ${ }^{23 b}$ Y. J. Mao, ${ }^{38, k}$ Z. P. Mao, ${ }^{1}$ S. Marcello, ${ }^{66 a, 66 c}$ Z. X. Meng, ${ }^{57}$ J. G. Messchendorp,${ }^{55}$

G. Mezzadri, ${ }^{24 a}$ T. J. Min, ${ }^{35}$ R. E. Mitchell, ${ }^{22}$ X. H. Mo,${ }^{1,49,54}$ Y. J. Mo, ${ }^{6}$ N. Yu. Muchnoi, ${ }^{10, c}$ H. Muramatsu, ${ }^{59}$

S. Nakhoul, ${ }^{11, \mathrm{f}}$ Y. Nefedov, ${ }^{29}$ F. Nerling, ${ }^{11, \mathrm{f}}$ I. B. Nikolaev, ${ }^{10, \mathrm{c}}$ Z. Ning, ${ }^{1,49}$ S. Nisar, ${ }^{8, \mathrm{i}}$ S. L. Olsen, ${ }^{54}$ Q. Ouyang, ${ }^{1,49,54}$ S. Pacetti, ${ }^{23 b, 23 c}$ X. Pan, ${ }^{9, h}$ Y. Pan ${ }^{58}$ A. Pathak, ${ }^{1}$ P. Patteri, ${ }^{23 a}$ M. Pelizaeus,${ }^{4}$ H. P. Peng, ${ }^{63,49}$ K. Peters, ${ }^{11, f}$ J. Pettersson, ${ }^{67}$ J. L. Ping, ${ }^{34}$ R. G. Ping, ${ }^{1,54}$ R. Poling,${ }^{59}$ V. Prasad, ${ }^{63,49}$ H. Qi, ${ }^{63,49}$ H. R. Qi ${ }^{52}$ K. H. Qi, ${ }^{25}$ M. Qi, ${ }^{35}$ T. Y. Qi ${ }^{9}$ T. Y. Qi, ${ }^{2}$ S. Qian, ${ }^{1,49}$ W. B. Qian, ${ }^{54}$ Z. Qian, ${ }^{50}$ C. F. Qiao, ${ }^{54}$ L. Q. Qin, ${ }^{12}$ X. P. Qin, ${ }^{9}$ X. S. Qin, ${ }^{41}$ Z. H. Qin, ${ }^{1,49}$ J. F. Qiu, ${ }^{1}$ S. Q. Qu ${ }^{36}$ K. Ravindran, ${ }^{21}$ C. F. Redmer, ${ }^{28}$ A. Rivetti, ${ }^{66}$ V. Rodin, ${ }^{55}$ M. Rolo, ${ }^{66 c}$ G. Rong, ${ }^{154}$ Ch. Rosner, ${ }^{15}$ M. Rump,${ }^{60}$ H. S. Sang, ${ }^{63}$ A. Sarantsev, ${ }^{29, d}$ Y. Schelhaas, ${ }^{28}$ C. Schnier, ${ }^{4}$ K. Schoenning, ${ }^{67}$ M. Scodeggio, ${ }^{24 a, 24 b}$ D. C. Shan, ${ }^{46}$ W. Shan, ${ }^{19}$

X. Y. Shan,${ }^{63,49}$ J. F. Shangguan, ${ }^{46}$ M. Shao,${ }^{63,49}$ C. P. Shen, ${ }^{9}$ P. X. Shen,${ }^{36}$ X. Y. Shen,${ }^{1,54}$ H. C. Shi, ${ }^{63,49}$ R. S. Shi, ${ }^{1,54}$ X. Shi, ${ }^{1,49}$ X. D. Shi, ${ }^{63,49}$ J. J. Song, ${ }^{41}$ W. M. Song, ${ }^{27,1}$ Y. X. Song, ${ }^{38, k}$ S. Sosio, ${ }^{66,66 c}$ S. Spataro, ${ }^{66,66 c}$ K. X. Su, ${ }^{68}$ P. P. Su, ${ }^{46}$ F. F. Sui ${ }^{41}$ G. X. Sun, ${ }^{1}$ H. K. Sun, ${ }^{1}$ J. F. Sun, ${ }^{16}$ L. Sun, ${ }^{68}$ S. S. Sun, ${ }^{1,54}$ T. Sun, ${ }^{1,54}$ W. Y. Sun ${ }^{34}$ W. Y. Sun, ${ }^{27}$ X. Sun ${ }^{20,1}$ Y. J. Sun,${ }^{63,49}$ Y. K. Sun ${ }^{63,49}$ Y.Z. Sun, ${ }^{1}$ Z. T. Sun, ${ }^{1}$ Y. H. Tan,${ }^{68}$ Y. X. Tan ${ }^{63,49}$ C. J. Tang, ${ }^{45}$ G. Y. Tang, ${ }^{1}$ J. Tang, ${ }^{50}$ J. X. Teng, ${ }^{63,49}$ V. Thoren, ${ }^{67}$ Y. T. Tian, ${ }^{25}$ I. Uman, ${ }^{53 b}$ B. Wang, ${ }^{1}$ C. W. Wang, ${ }^{35}$ D. Y. Wang, ${ }^{38, k}$ H. J. Wang,${ }^{31}$ H. P. Wang, ${ }^{1,54}$

K. Wang, ${ }^{1,49}$ L. L. Wang, ${ }^{1}$ M. Wang, ${ }^{41}$ M. Z. Wang, ${ }^{38, k}$ Meng Wang, ${ }^{1,54}$ W. Wang, ${ }^{50}$ W. H. Wang ${ }^{68}$ W. P. Wang, ${ }^{6,49}$

X. Wang, ${ }^{38, k}$ X. F. Wang, ${ }^{31}$ X. L. Wang, ${ }^{9, h}$ Y. Wang, ${ }^{63,49}$ Y. Wang, ${ }^{50}$ Y. D. Wang, ${ }^{37}$ Y. F. Wang, ${ }^{1,49,54}$ Y. Q. Wang, ${ }^{1}$ Y. Y. Wang, ${ }^{31}$ Z. Wang, ${ }^{1,49}$ Z. Y. Wang, ${ }^{1}$ Ziyi Wang, ${ }^{54}$ Zongyuan Wang ${ }^{1,54}$ D. H. Wei, ${ }^{12}$ P. Weidenkaff,${ }^{28}$ F. Weidner, ${ }^{60}$ S. P. Wen, ${ }^{1}$ D. J. White, ${ }^{58}$ U. Wiedner, ${ }^{4}$ G. Wilkinson, ${ }^{61}$ M. Wolke, ${ }^{67}$ L. Wollenberg, ${ }^{4}$ J. F. Wu,${ }^{1,54}$ L. H. Wu ${ }^{1}$ L. J. Wu, ${ }^{1,54}$ X. Wu, ${ }^{9, h}$ Z. Wu, ${ }^{1,49}$ L. Xia, ${ }^{63,49}$ H. Xiao, ${ }^{9, h}$ S. Y. Xiao, ${ }^{1}$ Z. J. Xiao, ${ }^{34}$ X. H. Xie, ${ }^{38, k}$ Y. G. Xie ${ }^{1,49}$ Y. H. Xie, ${ }^{6}$ T. Y. Xing, ${ }^{1,54}$

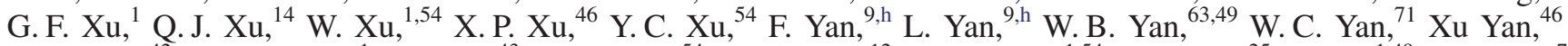
H. J. Yang, ${ }^{42,9}$ H. X. Yang, ${ }^{1}$ L. Yang, ${ }^{43}$ S. L. Yang, ${ }^{54}$ Y. X. Yang, ${ }^{12}$ Yifan Yang, ${ }^{1,54}$ Zhi Yang, ${ }^{25}$ M. Ye ${ }^{1,49}$ M. H. Ye, ${ }^{7}$ J. H. Yin, ${ }^{1}$ Z. Y. You, ${ }^{50}$ B. X. Yu, ${ }^{1,49,54}$ C. X. Yu, ${ }^{36}$ G. Yu, ${ }^{1,54}$ J. S. Yu, ${ }^{20,1}$ T. Yu, ${ }^{64}$ C. Z. Yuan, ${ }^{1,54}$ L. Yuan, ${ }^{2}$ X. Q. Yuan, ${ }^{38, k}$ Y. Yuan, ${ }^{1}$ Z. Y. Yuan, ${ }^{50}$ C. X. Yue, ${ }^{32}$ A. Yuncu,${ }^{53 a, a}$ A. A. Zafar, ${ }^{65}$ Y. Zeng, ${ }^{20,1}$ B. X. Zhang, ${ }^{1}$ Guangyi Zhang, ${ }^{16}$ H. Zhang, ${ }^{63}$ 
H. H. Zhang, ${ }^{50}$ H. H. Zhang, ${ }^{27}$ H. Y. Zhang, ${ }^{1,49}$ J. J. Zhang, ${ }^{43}$ J. L. Zhang, ${ }^{69}$ J. Q. Zhang, ${ }^{34}$ J. W. Zhang, ${ }^{1,49,54}$ J. Y. Zhang, ${ }^{1}$ J. Z. Zhang, ${ }^{1,54}$ Jianyu Zhang, ${ }^{1,54}$ Jiawei Zhang, ${ }_{1,54}$ L. M. Zhang, ${ }^{52}$ L. Q. Zhang, ${ }^{50}$ Lei Zhang, ${ }^{35}$ S. Zhang, ${ }^{50}$ S. F. Zhang, ${ }^{35}$ Shulei Zhang, ${ }^{20,1}$ X. D. Zhang, ${ }^{37}$ X. Y. Zhang, ${ }^{41}$ Y. Zhang, ${ }^{61}$ Y. H. Zhang, ${ }^{1,49}$ Y. T. Zhang, ${ }^{63,49}$ Yan Zhang, ${ }^{63,49}$ Yao Zhang, Yi Zhang, ${ }^{9,4}$ Z. H. Zhang, ${ }^{6}$ Z. Y. Zhang, ${ }^{68}$ G. Zhao, ${ }^{1}$ J. Zhao, ${ }^{32}$ J. Y. Zhao, ${ }^{1,54}$ J. Z. Zhao, ${ }^{1,49}$ Lei Zhao, ${ }^{63,49}$ Ling Zhao, ${ }^{1}$ M. G. Zhao, ${ }^{36}$ Q. Zhao, ${ }^{1}$ S. J. Zhao, ${ }^{71}$ Y. B. Zhao, ${ }^{1,49}$ Y. X. Zhao, ${ }^{25}$ Z. G. Zhao, ${ }^{63,49}$ A. Zhemchugov, ${ }^{29, b}$ B. Zheng, ${ }^{64}$ J. P. Zheng, ${ }^{1,49}$ Y. Zheng, ${ }^{38, k}$ Y. H. Zheng, ${ }^{54}$ B. Zhong, ${ }^{34}$ C. Zhong, ${ }^{64}$ L. P. Zhou, ${ }^{1,54}$ Q. Zhou, ${ }^{1,54}$ X. Zhou, ${ }^{68}$ X. K. Zhou, ${ }^{54}$ X. R. Zhou, ${ }^{63,49}$ A. N. Zhu, ${ }^{1,54}$ J. Zhu, ${ }^{36}$ K. Zhu, ${ }^{1}$ K. J. Zhu, ${ }^{1,49,54}$ S. H. Zhu, ${ }^{62}$ T. J. Zhu, ${ }^{69}$ W. J. Zhu, ${ }^{9}$, W. J. Zhu, ${ }^{36}$ Y. C. Zhu, ${ }^{63,49}$ Z. A. Zhu, ${ }^{1,54}$ B. S. Zou, ${ }^{1}$ and J. H. Zou ${ }^{1}$

\title{
(BESIII Collaboration)
}

\author{
${ }^{1}$ Institute of High Energy Physics, Beijing 100049, People's Republic of China \\ ${ }^{2}$ Beihang University, Beijing 100191, People's Republic of China \\ ${ }^{3}$ Beijing Institute of Petrochemical Technology, Beijing 102617, People's Republic of China \\ ${ }^{4}$ Bochum Ruhr-University, D-44780 Bochum, Germany \\ ${ }^{5}$ Carnegie Mellon University, Pittsburgh, Pennsylvania 15213, USA \\ ${ }^{6}$ Central China Normal University, Wuhan 430079, People's Republic of China \\ ${ }^{7}$ China Center of Advanced Science and Technology, Beijing 100190, People's Republic of China \\ ${ }^{8}$ COMSATS University Islamabad, Lahore Campus, Defence Road, Off Raiwind Road, \\ 54000 Lahore, Pakistan \\ ${ }^{9}$ Fudan University, Shanghai 200443, People's Republic of China \\ ${ }^{10}$ G.I. Budker Institute of Nuclear Physics SB RAS (BINP), Novosibirsk 630090, Russia \\ ${ }^{11}$ GSI Helmholtzcentre for Heavy Ion Research GmbH, D-64291 Darmstadt, Germany \\ ${ }^{12}$ Guangxi Normal University, Guilin 541004, People's Republic of China \\ ${ }^{13}$ Guangxi University, Nanning 530004, People's Republic of China \\ ${ }^{14}$ Hangzhou Normal University, Hangzhou 310036, People's Republic of China \\ ${ }^{15}$ Helmholtz Institute Mainz, Johann-Joachim-Becher-Weg 45, D-55099 Mainz, Germany \\ ${ }^{16}$ Henan Normal University, Xinxiang 453007, People's Republic of China \\ ${ }^{17}$ Henan University of Science and Technology, Luoyang 471003, People's Republic of China \\ ${ }^{18}$ Huangshan College, Huangshan 245000, People's Republic of China \\ ${ }^{19}$ Hunan Normal University, Changsha 410081, People's Republic of China \\ ${ }^{20}$ Hunan University, Changsha 410082, People's Republic of China \\ ${ }^{21}$ Indian Institute of Technology Madras, Chennai 600036, India \\ ${ }^{22}$ Indiana University, Bloomington, Indiana 47405, USA \\ ${ }^{23 a}$ INFN Laboratori Nazionali di Frascati, I-00044 Frascati, Italy \\ ${ }^{23 \mathrm{~b}}$ INFN Sezione di Perugia, I-06100 Perugia, Italy \\ ${ }^{23 \mathrm{c}}$ University of Perugia, I-06100 Perugia, Italy \\ ${ }^{24 a}$ INFN Sezione di Ferrara, I-44122 Ferrara, Italy \\ ${ }^{24 \mathrm{~b}}$ University of Ferrara, I-44122 Ferrara, Italy \\ ${ }^{25}$ Institute of Modern Physics, Lanzhou 730000, People's Republic of China \\ ${ }^{26}$ Institute of Physics and Technology, Peace Avenue 54B, Ulaanbaatar 13330, Mongolia \\ ${ }^{27}$ Jilin University, Changchun 130012, People's Republic of China \\ ${ }^{28}$ Johannes Gutenberg University of Mainz, Johann-Joachim-Becher-Weg 45, D-55099 Mainz, Germany \\ ${ }^{29}$ Joint Institute for Nuclear Research, 141980 Dubna, Moscow region, Russia \\ ${ }^{30}$ Justus-Liebig-Universitaet Giessen, II. Physikalisches Institut, Heinrich-Buff-Ring 16, \\ D-35392 Giessen, Germany \\ ${ }^{31}$ Lanzhou University, Lanzhou 730000, People's Republic of China \\ ${ }^{32}$ Liaoning Normal University, Dalian 116029, People's Republic of China \\ ${ }^{33}$ Liaoning University, Shenyang 110036, People's Republic of China \\ ${ }^{34}$ Nanjing Normal University, Nanjing 210023, People's Republic of China \\ ${ }^{35}$ Nanjing University, Nanjing 210093, People's Republic of China \\ ${ }^{36}$ Nankai University, Tianjin 300071, People's Republic of China \\ ${ }^{37}$ North China Electric Power University, Beijing 102206, People's Republic of China \\ ${ }^{38}$ Peking University, Beijing 100871, People's Republic of China \\ ${ }^{39}$ Qufu Normal University, Qufu 273165, People's Republic of China \\ ${ }^{40}$ Shandong Normal University, Jinan 250014, People's Republic of China \\ ${ }^{41}$ Shandong University, Jinan 250100, People's Republic of China \\ ${ }^{42}$ Shanghai Jiao Tong University, Shanghai 200240, People's Republic of China \\ ${ }^{43}$ Shanxi Normal University, Linfen 041004, People's Republic of China
}




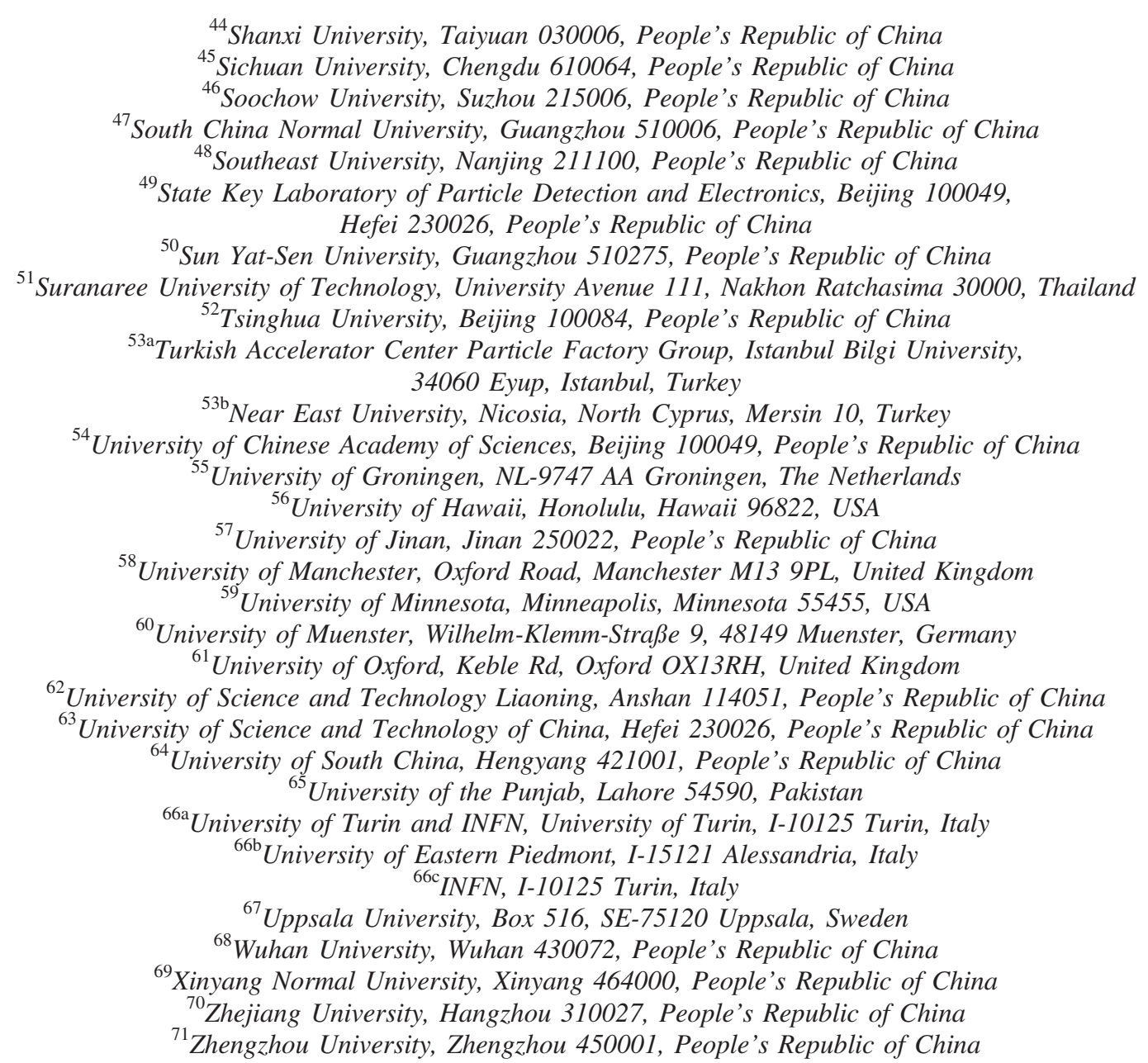

(Received 9 February 2021; accepted 9 April 2021; published 24 May 2021)

Using $6.32 \mathrm{fb}^{-1}$ of $e^{+} e^{-}$collision data collected by the BESIII detector at the center-of-mass energies between 4.178 and $4.226 \mathrm{GeV}$, an amplitude analysis of the $D_{s}^{+} \rightarrow K_{S}^{0} K^{-} \pi^{+} \pi^{+}$decays is performed for the first time to determine the intermediate-resonant contributions. The dominant component is the $D_{s}^{+} \rightarrow$ $K^{*}(892)^{+} \bar{K}^{*}(892)^{0}$ decay with a fraction of $\left(40.6 \pm 2.9_{\text {stat }} \pm 4.9_{\text {sys }}\right) \%$. Our results of the amplitude

${ }^{a}$ Also at Bogazici University, 34342 Istanbul, Turkey.

${ }^{\mathrm{b}}$ Also at the Moscow Institute of Physics and Technology, Moscow 141700, Russia.

${ }^{\mathrm{c}}$ Also at the Novosibirsk State University, Novosibirsk 630090, Russia.

dAlso at the NRC "Kurchatov Institute," PNPI, Gatchina 188300, Russia.

${ }^{\mathrm{e} A}$ Also at Istanbul Arel University, 34295 Istanbul, Turkey.

${ }^{\mathrm{f}}$ Also at Goethe University Frankfurt, 60323 Frankfurt am Main, Germany.

${ }^{\mathrm{g}}$ Also at Key Laboratory for Particle Physics, Astrophysics and Cosmology, Ministry of Education; Shanghai Key Laboratory for Particle Physics and Cosmology; Institute of Nuclear and Particle Physics, Shanghai 200240, People's Republic of China.

${ }^{\mathrm{h}}$ Also at Key Laboratory of Nuclear Physics and Ion-beam Application (MOE) and Institute of Modern Physics, Fudan University, Shanghai 200443, People's Republic of China.

${ }^{i}$ Also at Harvard University, Department of Physics, Cambridge, Massachusetts 02138, USA.

${ }^{\mathrm{j}}$ Currently at: Institute of Physics and Technology, Peace Avenue 54B, Ulaanbaatar 13330, Mongolia.

${ }^{\mathrm{k}}$ Also at State Key Laboratory of Nuclear Physics and Technology, Peking University, Beijing 100871, People's Republic of China. ${ }^{1}$ School of Physics and Electronics, Hunan University, Changsha 410082, China.

${ }^{\mathrm{m}}$ Also at Guangdong Provincial Key Laboratory of Nuclear Science, Institute of Quantum Matter, South China Normal University, Guangzhou 510006, China.

Published by the American Physical Society under the terms of the Creative Commons Attribution 4.0 International license. Further distribution of this work must maintain attribution to the author(s) and the published article's title, journal citation, and DOI. Funded by $S C O A P^{3}$. 
analysis are used to obtain a more precise measurement of the branching fraction of the $D_{s}^{+} \rightarrow K_{S}^{0} K^{-} \pi^{+} \pi^{+}$ decay, which is determined to be $\left(1.46 \pm 0.05_{\text {stat }} \pm 0.05_{\text {sys }}\right) \%$.

DOI: 10.1103/PhysRevD.103.092006

\section{INTRODUCTION}

The decay $D_{s}^{+} \rightarrow K_{S}^{0} K^{-} \pi^{+} \pi^{+}$is usually used as a "tag mode" for measurements related to the $D_{s}^{+}$meson [1-5] due to its large branching fraction and low background contamination. The inclusion of charge-conjugate states is implied throughout the paper. In 2013, the CLEO Collaboration reported its branching fraction $\mathcal{B}\left(D_{s}^{+} \rightarrow\right.$ $\left.K_{S}^{0} K^{-} \pi^{+} \pi^{+}\right)$to be $(1.64 \pm 0.07 \pm 0.08) \%$, based on a data sample corresponding to an integrated luminosity of $586 \mathrm{pb}^{-1}$ of $e^{+} e^{-}$collisions at a center-of-mass energy $\left(E_{\mathrm{cm}}\right)$ of $4.17 \mathrm{GeV}$ [6]. The measurement was limited by the sample size and lack of knowledge of the intermediate processes. In addition, the branching fraction of $D_{s}^{+} \rightarrow K^{*}(892)^{+} \bar{K}^{*}(892)^{0}$ was determined by the ARGUS Collaboration [7] more than twenty years ago, who claimed the contribution of $D_{s}^{+} \rightarrow K^{*}(892)^{+} \bar{K}^{*}(892)^{0}$ in the $D_{s}^{+} \rightarrow K_{S}^{0} K^{-} \pi^{+} \pi^{+}$decays is almost $100 \%$. The ARGUS measurement suffers from low statistics and large uncertainties in the branching fraction of the reference decay $D_{s}^{+} \rightarrow \phi(1020) \pi^{+}$. An amplitude analysis of the $D_{s}^{+} \rightarrow$ $K_{S}^{0} K^{-} \pi^{+} \pi^{+}$decays is necessary to investigate the resonant contributions, and thereby reduce the systematic uncertainties of its branching fraction and for providing input to measurements where amplitude information is essential.

It is well known that two-body modes dominate $D_{s}^{+}$ decays [8]. The majority of the observed two-body decays have pseudoscalar-pseudoscalar or pseudoscalar-vector mesons in the final states. Among various kinds of $D_{s}^{+}$decay modes, vector-vector final states are of special interest. The ratios between different orbital angular momenta of the two vector mesons for the dominant quasi-two-body decay $D_{s}^{+} \rightarrow K^{*}(892)^{+} \bar{K}^{*}(892)^{0}$ provide valuable information on $C P$ violation with T-violating triple products [9]. In addition, several mesons with $J^{P}=0^{-}, 1^{+}$are reported in the mass region between 1.2 and $1.6 \mathrm{GeV} / c^{2}$ and decay to the $(K \bar{K} \pi)^{0}$ final state [10-13]. These are the $\eta(1295), \eta(1405)$, $\eta(1475), f_{1}(1285), f_{1}(1420)$, and $f_{1}(1510)$, although many of these states are not well established.

This paper presents the first amplitude analysis and an improved branching-fraction measurement of the $D_{s}^{+} \rightarrow$ $K_{S}^{0} K^{-} \pi^{+} \pi^{+}$decay with data samples corresponding to a total integrated luminosity of $6.32 \mathrm{fb}^{-1}$ collected by the BESIII detector at $E_{\mathrm{cm}}$ between 4.178 and $4.226 \mathrm{GeV}$.

\section{DETECTOR AND DATA SETS}

The detailed description of the BESIII detector can be found in Ref. [14]. It is a magnetic spectrometer located at the Beijing Electron Positron Collider (BEPCII) [15]. The cylindrical core of the BESIII detector consists of a helium-based multilayer drift chamber (MDC), a plastic scintillator time-of-flight system (TOF), and a CsI (Tl) electromagnetic calorimeter (EMC), which are all enclosed in a superconducting solenoidal magnet providing a $1.0 \mathrm{~T}$ magnetic field. The solenoid is supported by an octagonal flux-return yoke with resistive plate counter muon-identifier modules interleaved with steel. The acceptance of charged particles and photons is $93 \%$ over the $4 \pi$ solid angle. The charged-particle momenta resolution at $1.0 \mathrm{GeV} / c$ is $0.5 \%$, and the specific energy loss $(d E / d x)$ resolution is $6 \%$ for the electrons from Bhabha scattering. The EMC measures photon energies with a resolution of $2.5 \%(5 \%)$ at $1 \mathrm{GeV}$ in the barrel (endcap) region. The time resolution of the TOF barrel part is $68 \mathrm{ps}$, while that of the end cap part is $110 \mathrm{ps}$. The end-cap TOF was upgraded in 2015 with multigap resistive plate chamber technology, providing a time resolution of 60 ps [16].

The data samples used in this paper were accumulated in the years 2013, 2016, and 2017 with $E_{\mathrm{cm}}$ of 4.226, 4.178, and 4.189-4.219 GeV, respectively. Generic Monte Carlo (GMC) samples that are 40 times larger than the data sets are produced with the GEANT4-based software [17]. The production of open-charm processes directly via $e^{+} e^{-}$ annihilation is modeled with the generator CONEXC [18], which includes the effects of the beam energy spread and initial state radiation (ISR). The ISR production of vector charmonium states and the continuum processes are incorporated in KKMC [19]. The known decay modes are generated using EVTGEN [20], which assumes the branching fractions reported by the Particle Data Group (PDG) [8]. The remaining unknown decays from the charmonium states are generated with LUNDCHARM [21]. The final state radiation from charged tracks are simulated by the PнOTOS package [22]. The GMC is used to estimate background and optimize selection criteria.

More than 10 million simulated events are generated with a uniform distribution in the phase space of the $D_{s}^{+} \rightarrow K_{S}^{0} K^{-} \pi^{+} \pi^{+}$decay to perform the normalization in the amplitude fit. Preliminary parameters of the amplitude model are obtained from an initial fit to the data. A signal Monte Carlo (SMC) sample is generated according to the preliminary parameters and is used to validate the fit performance and to estimate the detector efficiency. A final determination of the fit parameters is obtained by fitting the data using the SMC sample for the normalization. 


\section{EVENT SELECTIONS}

The production of $D_{s}^{ \pm}$candidates is dominated by the process $e^{+} e^{-} \rightarrow D_{s}^{*+} D_{s}^{-}$, where the $D_{s}^{*+}$ meson decays to either $\gamma D_{s}^{+}$or $\pi^{0} D_{s}^{+}$with branching fractions of $(93.5 \pm$ $0.7) \%$ and $(5.8 \pm 0.7) \%$ [8], respectively. A sample of $D_{s}^{-}$ mesons is reconstructed first, with nine $D_{s}^{-}$prominent hadronic decay modes, as shown in Table $\mathrm{I}$, and is referred to as the "single tag (ST)" candidates. The signal decay $D_{s}^{+} \rightarrow K_{S}^{0} K^{-} \pi^{+} \pi^{+}$is reconstructed by selecting two $\pi^{+}$, one $K^{-}$and one $K_{S}^{0}$ candidates from the unused tracks in each ST event, and is referred to as the sample of "double tag (DT)" candidates.

All charged tracks reconstructed in the MDC must satisfy $|\cos \theta|<0.93$, where $\theta$ is the polar angle with respect to the direction of the positron beam. Except for $K_{S}^{0}$ daughters, they must originate from the interaction point with a distance of closest approach less than $1 \mathrm{~cm}$ in the transverse plane and less than $10 \mathrm{~cm}$ along the beam direction. The $d E / d x$ information in the MDC and the time-of-fight information from the TOF are combined and used for particle identification (PID) by forming confidence levels $C L_{K(\pi)}$ for kaon (pion) hypotheses. Kaon (pion) candidates are required to satisfy $C L_{K(\pi)}>C L_{\pi(K)}$.

For the photon identification, it is required that each electromagnetic shower starts within $700 \mathrm{~ns}$ of the event start time and its energy is greater than 25 (50) $\mathrm{MeV}$ in the barrel (end cap) with $|\cos \theta|<0.80$ $(|\cos \theta| \in[0.86,0.92])$. The $\pi^{0}$ and $\eta$ candidates are reconstructed via diphoton decays $\left(\pi^{0} / \eta \rightarrow \gamma \gamma\right)$ with the invariant mass of the $\gamma \gamma$ combination $M_{\gamma \gamma} \in[0.115,0.150]$

TABLE I. The requirements of $M_{\text {rec }}$ for various energies and $M_{\text {tag }}$ for individual single-tagged modes. The $K_{S}^{0}, \pi^{0}(\eta)$, and $\eta^{\prime}$ mesons decay to $\pi^{+} \pi^{-}, \gamma \gamma$, and $\pi^{+} \pi^{-} \eta$ final states, respectively.

\begin{tabular}{lc}
\hline \hline$E_{\mathrm{cm}}(\mathrm{GeV})$ & $M_{\mathrm{rec}}\left(\mathrm{GeV} / c^{2}\right)$ \\
\hline 4.178 & {$[2.050,2.180]$} \\
4.189 & {$[2.048,2.190]$} \\
4.199 & {$[2.046,2.200]$} \\
4.209 & {$[2.044,2.210]$} \\
4.219 & {$[2.042,2.220]$} \\
4.226 & {$[2.040,2.220]$} \\
\hline \hline
\end{tabular}

\begin{tabular}{lr}
\hline \hline Tag mode & $M_{\operatorname{tag}}\left(\mathrm{GeV} / c^{2}\right)$ \\
\hline$D_{s}^{-} \rightarrow K_{S}^{0} K^{-}$ & {$[1.948,1.991]$} \\
$D_{s}^{-} \rightarrow K^{+} K^{-} \pi^{-}$ & {$[1.950,1.986]$} \\
$D_{s}^{-} \rightarrow K_{S}^{0} K^{-} \pi^{0}$ & {$[1.946,1.987]$} \\
$D_{s}^{-} \rightarrow K^{-} \pi^{+} \pi^{-}$ & {$[1.953,1.983]$} \\
$D_{s}^{-} \rightarrow \pi^{-} \eta^{\prime}$ & {$[1.940,1.996]$} \\
$D_{s}^{-} \rightarrow K_{S}^{0} K^{-} \pi^{+} \pi^{-}$ & {$[1.958,1.980]$} \\
$D_{s}^{-} \rightarrow K^{+} K^{-} \pi^{-} \pi^{0}$ & {$[1.947,1.982]$} \\
$D_{s}^{-} \rightarrow \pi^{+} \pi^{-} \pi^{-}$ & {$[1.952,1.982]$} \\
$D_{s}^{-} \rightarrow \pi^{-} \eta$ & {$[1.930,2.000]$} \\
\hline \hline
\end{tabular}

and $[0.50,0.57] \mathrm{GeV} / c^{2}$, respectively. The value of $M_{\gamma \gamma}$ is constrained to the $\pi^{0}$ or $\eta$ nominal mass [8] by a kinematic fit, and the $\chi^{2}$ of the kinematic fit must be less than 30 . We reconstruct the $\eta^{\prime} \rightarrow \pi^{+} \pi^{-} \eta$ candidates by requiring $M_{\pi^{+} \pi^{-} \eta} \in[0.946,0.970] \mathrm{GeV} / c^{2}$.

The $K_{S}^{0}$ candidates are selected by looping over all pairs of tracks with opposite charges, whose distances to the interaction point along the beam direction are within $20 \mathrm{~cm}$. A primary vertex and a secondary vertex [23] are reconstructed and the decay length between the two is required to be greater than twice its uncertainty. Since the combinatorial background is low, this requirement is not applied for the $D_{s}^{-} \rightarrow K_{S}^{0} K^{-}$decay. The invariant mass $M_{\pi^{+} \pi^{-}}$is required to be in the region $[0.487,0.511] \mathrm{GeV} / c^{2}$. To prevent an event being retained by both the $D_{s}^{-} \rightarrow K_{S}^{0} K^{-}$and $D_{s}^{-} \rightarrow K^{-} \pi^{+} \pi^{-}$selections, the value of $M_{\pi^{+} \pi^{-}}$is required to be outside of the mass range $[0.487,0.511] \mathrm{GeV} / c^{2}$ for the $D_{s}^{-} \rightarrow K^{-} \pi^{+} \pi^{-}$ decay.

\section{AMPLITUDE ANALYSIS}

\section{A. Selections for amplitude analysis}

The tagged $D_{s}^{-}$candidates are constructed from the $\pi^{+}$, $K^{-}, \eta, \eta^{\prime}, K_{S}^{0}$, and $\pi^{0}$ mesons, while the signal $D_{s}^{+}$ candidates are reconstructed from the $K_{S}^{0}, K^{-}$, and two $\pi^{+}$mesons. The requirements on the recoiling mass of the $D_{s}^{+}, M_{\text {rec }}$, and the mass of the tagged $D_{s}^{-}, M_{\text {tag }}$, are summarized in Table I. The recoiling mass is calculated as follows:

$$
M_{\mathrm{rec}}=\sqrt{\left(E_{\mathrm{cm}}-\sqrt{\overrightarrow{\mathbf{p}}_{D_{s}^{+}}^{2}+m_{D_{s}^{+}}^{2}}\right)^{2}-\overrightarrow{\mathbf{p}}_{D_{s}^{+}}^{2}} .
$$

Here, $\overrightarrow{\mathbf{p}}_{D_{s}^{+}}$is the three-momentum of the $D_{s}^{+}$candidate and $m_{D_{s}^{+}}$is its nominal mass [8].

Kinematic fits are performed of the process $e^{+} e^{-} \rightarrow$ $D_{s}^{* \pm} D_{s}^{\mp} \rightarrow \gamma D_{s}^{ \pm} D_{s}^{\mp}$ with the photon assigned to each charm meson in turn, and the $\chi^{2}$ of the fit being used to decide between the $D_{s}^{*+}$ and $D_{s}^{*-}$ hypotheses. The fits include constraints from four-momentum conservation in the $e^{+} e^{-}$system and also constrain the invariant masses of $K_{S}^{0}, D_{s}^{* \pm}$, and tag-side $D_{s}^{ \pm}$candidates to their nominal masses [8]. In order to ensure that all candidates fall within the kinematic boundary of the phase space, we perform a further kinematic fit in which the signal $D_{s}^{ \pm}$mass is constrained to its nominal value, and the updated fourmomenta are used for the amplitude analysis.

To suppress the background where the $\pi^{-}$from the signal decay and the $\pi^{+}$from the tag modes are exchanged, which fakes the signal and the same tag mode but with opposite charges, we perform kinematic fits with $D_{s}^{+}$and $D_{s}^{-}$mass constraints for the two cases and select the one with the smaller $\chi^{2}$. To reduce the background coming from 

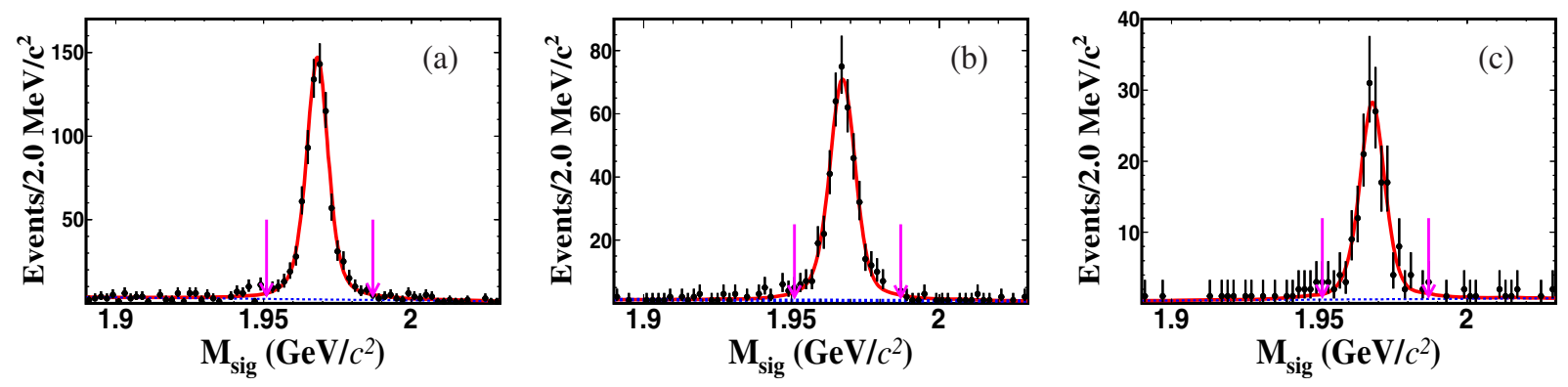

FIG. 1. Fits to the $M_{\text {sig }}$ distributions of accepted candidates from the data samples taken at (a) $E_{\text {cm }}=4.178$, (b) $4.189-4.219$, and (c) $4.226 \mathrm{GeV}$, respectively. The points with error bars are data. The red solid curves are the fit results. The blue dotted curves are the fitted background shapes. The pair of pink arrows indicate the chosen signal region.

$D^{0} \rightarrow K^{-} \pi^{+} \pi^{+} \pi^{-}$versus $\bar{D}^{0} \rightarrow K_{S}^{0} K^{+} K^{-}\left(K_{S}^{0} \pi^{+} \pi^{-}\right)$, by exchanging $\pi^{-}$from $D^{0}$ and $K_{S}^{0}$ from $\bar{D}^{0}$, faking the signal $D_{s}^{+} \rightarrow K_{S}^{0} K^{-} \pi^{+} \pi^{+}$and the tag mode $D_{s}^{-} \rightarrow K^{+} K^{-} \pi^{-}\left(\pi^{+} \pi^{-} \pi^{-}\right)$, we reject events satisfying $\left|M_{D^{0}}^{\prime}-M_{D^{0}}^{\mathrm{PDG}}\right|<15 \mathrm{MeV} / c^{2},\left|M_{\bar{D}^{0}}^{\prime}-M_{D^{0}}^{\mathrm{PDG}}\right|<15 \mathrm{MeV} / c^{2}$, and $\left|M_{D^{0}}^{\prime}-M_{\bar{D}^{0}}^{\prime}\right|<\left|M_{D_{s}^{+}}^{\prime}-M_{D_{s}^{-}}^{\prime}\right|$. Here, $M_{D^{0}}^{\mathrm{PDG}}$ is the nominal $D^{0}$ mass [8], $M_{D^{0}}^{\prime}, M_{\bar{D}^{0}}^{\prime}, M_{D_{s}^{+}}^{\prime}$, and $M_{D_{s}^{-}}^{\prime}$ are the invariant masses of the $D^{0} \rightarrow K^{-} \pi^{+} \pi^{+} \pi^{-}, \bar{D}^{0} \rightarrow$ $K_{S}^{0} K^{+} K^{-}\left(K_{S}^{0} \pi^{+} \pi^{-}\right), \quad D_{s}^{+} \rightarrow K_{S}^{0} K^{-} \pi^{+} \pi^{+}, \quad$ and $\quad D_{s}^{-} \rightarrow$ $K^{+} K^{-} \pi^{-}\left(\pi^{+} \pi^{-} \pi^{-}\right)$candidates, respectively.

Figure 1 shows the invariant mass distributions of the signal $D_{s}^{+}, M_{\text {sig }}$, in data and the fit results. The signal distribution is modeled with the simulated shape convolved with a Gaussian function and the background is described by a first-order Chebychev polynomial. The fitted yields for the signal are $744 \pm 28,415 \pm 21$, and $159 \pm 13$ in the invariant mass range $[1.951,1.987] \mathrm{GeV} / c^{2}$, with purities of $(94.7 \pm 0.5) \%,(96.2 \pm 0.7) \%$, and $(93.9 \pm 1.2) \%$ for the data samples taken at $E_{c m}=4.178,4.189-4.219$, and $4.226 \mathrm{GeV}$, respectively. The candidates falling in the $D_{s}^{+}$ mass region are retained for the amplitude analysis. We compare the background yield and various distributions of the events outside the signal region between data and GMC. The yield and distributions are found to be consistent within the statistical uncertainties. The background events in the signal region from GMC are used to estimate the background contributions in data.

\section{B. Likelihood function}

An unbinned-maximum-likelihood method is applied to determine resonant contributions in the $D_{s}^{+} \rightarrow K_{S}^{0} K^{-} \pi^{+} \pi^{+}$ decays. The likelihood function is constructed with a probability density function (PDF) of the momenta of the four daughter particles. The amplitude of the $n$th intermediate state $\left(A_{n}\right)$ is

$$
A_{n}=P_{n}^{1} P_{n}^{2} S_{n} F_{n}^{1} F_{n}^{2} F_{n}^{3},
$$

where the indices 1,2 , and 3 correspond to the two subsequent intermediate resonances and the $D_{s}^{+}$meson.
$S$ is the spin factor constructed with the covariant tensor formalism [24], $F$ is the Blatt-Weisskopf barrier factor, and $P$ is the propagator of the intermediate resonance. The total amplitude $M$ is a coherent sum of the amplitudes of intermediate processes,

$$
M=\sum c_{n} A_{n},
$$

where $c_{n}=\rho_{n} e^{i \phi_{n}}$ are complex coefficients to be determined from the fit to data.

The signal $\operatorname{PDF} f_{S}\left(p_{j}\right)$ is given by

$$
f_{S}\left(p_{j}\right)=\frac{\epsilon\left(p_{j}\right)\left|M\left(p_{j}\right)\right|^{2} R_{4}\left(p_{j}\right)}{\int \epsilon\left(p_{j}\right)\left|M\left(p_{j}\right)\right|^{2} R_{4}\left(p_{j}\right) d p_{j}},
$$

where $\epsilon\left(p_{j}\right)$ is the detection efficiency parametrized in terms of the final four-momenta $p_{j}$ and $j$ refers to the different particles in the final states. $R_{4}\left(p_{j}\right)$ is the standard element of the four-body phase space.

The normalization is determined from the simulated events,

$$
\int \epsilon\left(p_{j}\right)\left|M\left(p_{j}\right)\right|^{2} R_{4}\left(p_{j}\right) d p_{j} \approx \frac{1}{N_{\text {sim }}} \sum_{k_{\text {sim }}}^{N_{\text {sim }}} \frac{\left|M\left(p_{j}^{k_{\text {sim }}}\right)\right|^{2}}{\left|M^{\text {gen }}\left(p_{j}^{k_{\text {sim }}}\right)\right|^{2}},
$$

where $k_{\text {sim }}$ runs from 1 to $N_{\text {sim }}$, the total number of simulated events. $M^{\text {gen }}\left(p_{j}\right)$ is the PDF used to generate the simulated samples.

The normalization takes into account the difference in detector efficiencies for PID and tracking between data and simulation by assigning a weight to each simulated event,

$$
\gamma_{\epsilon}(p)=\prod_{i} \frac{\epsilon_{i, \mathrm{data}}\left(p_{j}\right)}{\epsilon_{i, \operatorname{sim}}\left(p_{j}\right)},
$$

where $i$ denotes the four daughter particles. The normalization is then given by 


$$
\int \epsilon\left(p_{j}\right)\left|M\left(p_{j}\right)\right|^{2} R_{4}\left(p_{j}\right) d p_{j} \approx \frac{1}{N_{\text {sim }}} \sum_{k_{\text {sim }}}^{N_{\text {sim }}} \frac{\gamma_{\epsilon}\left(p_{j}^{k_{\text {sim }}}\right)\left|M\left(p_{j}^{k_{\text {sim }}}\right)\right|^{2}}{\left|M^{\operatorname{gen}}\left(p_{j}^{k_{\text {sim }}}\right)\right|^{2}} .
$$

The total PDF $f_{T}\left(p_{j}\right)$ is

$$
\begin{aligned}
f_{T}\left(p_{j}\right)= & w \frac{\epsilon\left(p_{j}\right)\left|M\left(p_{j}\right)\right|^{2} R_{4}\left(p_{j}\right)}{\int \epsilon\left(p_{j}\right)\left|M\left(p_{j}\right)\right|^{2} R_{4}\left(p_{j}\right) d p_{j}} \\
& +(1-w) \frac{B\left(p_{j}\right) R_{4}\left(p_{j}\right)}{\int B\left(p_{j}\right) R_{4}\left(p_{j}\right) d p_{j}},
\end{aligned}
$$

where $w$ is the purity of the signal described by a constant parameter in the fit. We factorize out $\epsilon\left(p_{j}\right)$ from $f_{T}\left(p_{j}\right)$ as $\epsilon\left(p_{j}\right)$ is independent of the fit variables. Its contribution enters into the normalization and the background PDF. As a consequence, the combined PDF becomes

$$
\begin{aligned}
f_{T}\left(p_{j}\right)= & \epsilon\left(p_{j}\right) R_{4}\left(p_{j}\right)\left[w \frac{\left|M\left(p_{j}\right)\right|^{2}}{\int \epsilon\left(p_{j}\right)\left|M\left(p_{j}\right)\right|^{2} R_{4}\left(p_{j}\right) d p_{j}}\right. \\
& \left.+(1-w) \frac{B_{\epsilon}\left(p_{j}\right)}{\int \epsilon\left(p_{j}\right) B_{\epsilon}\left(p_{j}\right) R_{4}\left(p_{j}\right) d p_{j}}\right],
\end{aligned}
$$

where $B_{\epsilon}\left(p_{j}\right)=B\left(p_{j}\right) / \epsilon$ and the background $\operatorname{PDF} B\left(p_{j}\right)$ is parametrized using RooNDKeysPdf [25]. The normalization in the denominator of the background term is calculated as

$$
\int \epsilon\left(p_{j}\right) B_{\epsilon}\left(p_{j}\right) R_{4}\left(p_{j}\right) d p_{j} \approx \frac{1}{N_{\text {sim }}} \sum_{k_{\text {sim }}}^{N_{\text {sim }}} \frac{B_{\epsilon}\left(p_{j}^{k_{\text {sim }}}\right)}{\left|M^{\text {gen }}\left(p_{j}^{k_{\text {sim }}}\right)\right|^{2}} .
$$

Finally, the log-likelihood is written as

$$
\begin{aligned}
\ln \mathcal{L}= & \ln \left[w \frac{\left|M\left(p_{j}\right)\right|^{2}}{\int \epsilon\left(p_{j}\right)\left|M\left(p_{j}\right)\right|^{2} R_{4}\left(p_{j}\right) d p_{j}}\right. \\
& \left.+(1-w) \frac{B_{\epsilon}\left(p_{j}\right)}{\int \epsilon\left(p_{j}\right) B_{\epsilon}\left(p_{j}\right) R_{4}\left(p_{j}\right) d p_{j}}\right],
\end{aligned}
$$

and data samples collected at different $E_{c m}$ are fitted simultaneously.

\section{Spin factors}

For the process $a \rightarrow b c$, the four-momenta of the particles $a, b$, and $c$ are denoted as $p_{a}, p_{b}$, and $p_{c}$, respectively. The spin projection operators [24] are defined as

$$
\begin{aligned}
P_{\mu \mu^{\prime}}^{(1)}(a)= & -g_{\mu \mu^{\prime}}+\frac{p_{a, \mu} p_{a, \mu^{\prime}}}{p_{a}^{2}}, \\
P_{\mu \nu \mu^{\prime} \nu^{\prime}}^{(2)}(a)= & \frac{1}{2}\left(P_{\mu \mu^{\prime}}^{(1)}(a) P_{\nu \nu^{\prime}}^{(1)}(a)+P_{\mu \nu^{\prime}}^{(1)}(a) P_{\nu \mu^{\prime}}^{(1)}(a)\right) \\
& -\frac{1}{3} P_{\mu \nu}^{(1)}(a) P_{\mu \mu^{\prime} \nu^{\prime}}^{(1)}(a) .
\end{aligned}
$$

TABLE II. The spin factor $S(p)$ for each decay chain. All operators, i.e., $\tilde{t}$, have the same definitions as in Ref. [24]. Scalar, pseudoscalar, vector, and axial-vector states are denoted by $S, P, V$, and $A$, respectively. The $[S],[P]$, and $[D]$ denote the orbital angular-momentum quantum numbers $L=0,1$, and 2 , respectively.

\begin{tabular}{lc}
\hline \hline Decay chain & $S(p)$ \\
\hline$D_{s}^{+}[S] \rightarrow V_{1} V_{2}$ & $\tilde{t}^{(1) \mu}\left(V_{1}\right) \tilde{t}_{\mu}^{(1)}\left(V_{2}\right)$ \\
$D_{s}^{+}[P] \rightarrow V_{1} V_{2}$ & $\epsilon_{\mu \nu \lambda \sigma} p^{\mu}\left(D_{s}^{+}\right) \tilde{T}^{(1) \nu}\left(D_{s}^{+}\right)$ \\
& $\times \tilde{t}^{(1) \lambda}\left(V_{1}\right) \tilde{t}^{(1) \sigma}\left(V_{2}\right)$ \\
$D_{s}^{+}[D] \rightarrow V_{1} V_{2}$ & $\tilde{T}^{(2) \mu \nu}\left(D_{s}^{+}\right) \tilde{t}_{\mu}^{(1)}\left(V_{1}\right) \tilde{t}_{\nu}^{(1)}\left(V_{2}\right)$ \\
$D_{s}^{+} \rightarrow A P_{1}, A[S] \rightarrow V P_{2}$ & $\tilde{T}^{(1) \mu}\left(D_{s}^{+}\right) P_{\mu \nu}^{(1)}(A) \tilde{t}^{(1) \nu}(V)$ \\
$D_{s}^{+} \rightarrow A P_{1}, A \rightarrow S P_{2}$ & $\tilde{T}^{(1) \mu}\left(D_{s}^{+}\right) \tilde{t}_{\mu}^{(1)}(A)$ \\
$D_{s}^{+} \rightarrow V S$ & $\tilde{T}^{(1) \mu}\left(D_{s}^{+} \tilde{t}_{\mu}^{(1)}(V)\right.$ \\
$D_{s}^{+} \rightarrow P P_{1}, P \rightarrow V P_{2}$ & $p^{\mu}\left(P_{2}\right) \tilde{t}_{\mu}^{(1)}(V)$ \\
$D_{s}^{+} \rightarrow P P_{1}, P \rightarrow S P_{2}$ & 1 \\
\hline \hline
\end{tabular}

The pure orbital angular-momentum covariant tensors are given by

$$
\begin{aligned}
& \tilde{t}_{\mu}^{(1)}(a)=-P_{\mu \mu^{\prime}}^{(1)}(a) r_{a}^{\mu^{\prime}}, \\
& \tilde{t}_{\mu \nu}^{(2)}(a)=P_{\mu \nu \mu^{\prime} \nu^{\prime}}^{(2)}(a) r_{a}^{\mu^{\prime}} r_{a}^{\nu^{\prime}},
\end{aligned}
$$

where $r_{a}=p_{b}-p_{c}$. The spin factors $S(p)$ used in this paper are constructed from the spin projection operators and pure orbital angular-momentum covariant tensors and are listed in Table II.

\section{Blatt-Weisskopf barrier factors}

For the process $a \rightarrow b c$, the Blatt-Weisskopf barrier factor $F_{L}\left(p_{j}\right)$ is parametrized as a function of the angular momentum $L$ and the momentum $q$ of the daughter $b$ or $c$ in the rest system of $a$,

$$
F_{L}(q)=z^{L} X_{L}(q)
$$

where $z=q R . R$ is the effective radius of the barrier, which is fixed to $3.0 \mathrm{GeV}^{-1} \times \hbar c$ for the intermediate resonances and $5.0 \mathrm{GeV}^{-1} \times \hbar c$ for the $D_{s}^{+}$meson [26]. The momentum transfer squared is

$$
q^{2}=\frac{\left(s_{a}+s_{b}-s_{c}\right)^{2}}{4 s_{a}}-s_{b},
$$

where $s_{a, b, c}$ are the invariant-mass squared of particles $a, b$, $c$, respectively. The $X_{L}(q)$ factors are given by

$$
\begin{aligned}
& X_{L=0}(q)=1 \\
& X_{L=1}(q)=\sqrt{\frac{2}{z^{2}+1}} \\
& X_{L=2}(q)=\sqrt{\frac{13}{z^{4}+3 z^{2}+9}} .
\end{aligned}
$$




\section{E. Propagators}

The propagators for the resonances $K^{*}(892)^{+}, \bar{K}^{*}(892)^{0}$, $\eta(1295), \eta(1405), \eta(1475), f_{1}(1285), f_{1}(1420)$, and $f_{1}(1510)$ are modeled by the relativistic Breit-Wigner function, which is given by

$$
\begin{aligned}
P(m) & =\frac{1}{\left(m_{0}^{2}-s_{a}\right)-i m_{0} \Gamma(m)}, \\
\Gamma(m) & =\Gamma_{0}\left(\frac{q}{q_{0}}\right)^{2 L+1}\left(\frac{m_{0}}{m}\right)\left(\frac{X_{L}(q)}{X_{L}\left(q_{0}\right)}\right)^{2},
\end{aligned}
$$

where $m_{0}$ and $\Gamma(m)$ are the mass and width of the intermediate resonance, and $q_{0}$ is the value of $q$ when $s_{a}=m_{0}^{2}$.

The $a_{0}(980)$ contribution is parametrized as the Flatté formula

$$
P_{a_{0}(980)}=\frac{1}{M^{2}-s_{a}-i\left(g_{\eta \pi} \rho_{\eta \pi}\left(s_{a}\right)+g_{K \bar{K}} \rho_{K \bar{K}}\left(s_{a}\right)\right)},
$$

where $\rho_{\eta \pi}\left(s_{a}\right)$ and $\rho_{K \bar{K}}\left(s_{a}\right)$ are the Lorentz-invariant phasespace factors defined as $2 q / \sqrt{s_{a}}$, and the coupling constants $g_{\eta \pi}^{2}=0.341 \pm 0.004 \mathrm{GeV}^{2} / c^{4}$ and $g_{K \bar{K}}^{2}=(0.892 \pm$ $0.022) g_{\eta \pi}^{2}[27]$.

We use the same parametrization to describe the $K \pi S$ wave as in Ref. [28], which is extracted from scattering data [29]. The model is built with a Breit-Wigner shape for the $K^{*}(1430)^{0}$ and an effective range parametrization for the nonresonant component,

$$
A(m)=F \sin \delta_{F} e^{i \delta_{F}}+R \sin \delta_{R} e^{i \delta_{R}} e^{i 2 \delta_{F}},
$$

with

TABLE III. The $\phi$, FFs and significances for different resonant contributions, labeled as I, II, III, .., XIII, respectively. The first and second uncertainties are the statistical and systematic uncertainties, respectively. Here $K^{*}(892)^{+}, \bar{K}^{*}(892)^{0}$, and $a_{0}(980)^{-}$denote $K^{*}(892)^{+} \rightarrow K_{S}^{0} \pi^{+}, \bar{K}^{*}(892)^{0} \rightarrow K^{-} \pi^{+}$, and $a_{0}(980)^{-} \rightarrow K_{S}^{0} K^{-}$, respectively, while $K(892)^{*} K$ indicates $\bar{K}^{* 0} K_{S}^{0}$ and $K^{*}(892)^{+} K^{-}$. The FF of IV (IIX) term is the sum of I, II, and III (VIII and IX) terms after considering the interference.

\begin{tabular}{lccrc}
\hline \hline Label & \multicolumn{1}{c}{ Component } & \multicolumn{1}{c}{$\phi$} & \multicolumn{1}{c}{$\mathrm{FF}(\%)$} & Significance $(\sigma)$ \\
\hline I & $D_{s}^{+}[S] \rightarrow K^{*}(892)^{+} \bar{K}^{*}(892)^{0}$ & 0 (fixed) & $34.3 \pm 3.1 \pm 5.2$ & $>10.0$ \\
II & $D_{s}^{+}[P] \rightarrow K^{*}(892)^{+} \bar{K}^{*}(892)^{0}-1.61$ & $0.08 \pm 0.03 \pm 7.5$ & $1.1 \pm 0.1 \pm 8.3$ & \\
III & $D_{s}^{+}[D] \rightarrow K^{*}(892)^{+} \bar{K}^{*}(892)^{0}$ & $-0.16 \pm 0.14 \pm 0.04$ & $4.5 \pm 0.8 \pm 0.3$ & 8.2 \\
IV & $D_{s}^{+} \rightarrow K^{*}(892)^{+} \bar{K}^{*}(892)^{0}$ & & $40.6 \pm 2.9 \pm 4.9$ & \\
V & $D_{s}^{+} \rightarrow K^{*}(892)^{+}\left(K^{-} \pi^{+}\right)_{S-\text { wave }}$ & $1.85 \pm 0.15 \pm 0.09$ & $5.0 \pm 1.2 \pm 1.0$ & 6.2 \\
VI & $D_{s}^{+} \rightarrow \bar{K}^{*}(892)^{0}\left(K_{S}^{0} \pi^{+}\right)_{S-\text { wave }}$ & $-1.57 \pm 0.12 \pm 0.13$ & $7.3 \pm 1.1 \pm 0.9$ & 9.1 \\
VII & $D_{s}^{+} \rightarrow \eta(1475) \pi^{+}, \eta(1475) \rightarrow a_{0}(980)^{-} \pi^{+}$ & $-1.95 \pm 0.15 \pm 0.07$ & $10.8 \pm 2.6 \pm 5.2$ & 4.4 \\
VIII & $D_{s}^{+} \rightarrow \eta(1475) \pi^{+}, \eta(1475) \rightarrow \bar{K}^{*}(892)^{0} K_{S}^{0}$ & $0.05 \pm 0.15 \pm 0.11$ & $2.2 \pm 0.6 \pm 0.2$ & 4.5 \\
IX & $D_{s}^{+} \rightarrow \eta(1475) \pi^{+}, \eta(1475) \rightarrow K^{*}(892)^{+} K^{-}$ & $0.05 \pm 0.15 \pm 0.11$ & $2.2 \pm 0.6 \pm 0.2$ & 4.5 \\
IIX & $D_{s}^{+} \rightarrow \eta(1475) \pi^{+}, \eta(1475) \rightarrow K^{*}(892) K_{1}$ & & $4.9 \pm 1.4 \pm 1.0$ & \\
IIIX & $D_{s}^{+} \rightarrow \eta(1475) \pi^{+}, \eta(1475) \rightarrow\left(K_{S}^{0} \pi^{+}\right)_{S-\text { wave }} K^{-}$ & $2.30 \pm 0.11 \pm 0.07$ & $23.6 \pm 3.6 \pm 7.5$ & 6.7 \\
X & $D_{s}^{+} \rightarrow f_{1}(1285) \pi^{+}, f_{1}(1285) \rightarrow a_{0}(980)^{-} \pi^{+}$ & $-0.89 \pm 0.26 \pm 0.14$ & $2.2 \pm 0.5 \pm 0.2$ & 6.0 \\
XI & $D_{s}^{+} \rightarrow\left(K^{*}(892)^{+} K^{-}\right)_{P} \pi^{+},\left(K^{*}(892)^{+} K^{-}\right)_{P} \rightarrow K^{*}(892)^{+} K^{-}$ & $-1.07 \pm 0.11 \pm 0.03$ & $10.8 \pm 1.9 \pm 1.7$ & 9.2 \\
\hline \hline
\end{tabular}

where the integration is approximated by the sum of the simulated events generated flatly over the phase space and without any efficiency effects included.

To estimate the statistical uncertainties on the FFs, the calculation is repeated by randomly varying the fit parameters according to the error matrix. The resulting distribution of each FF is fitted with a Gaussian function, whose width gives the corresponding statistical uncertainty. 

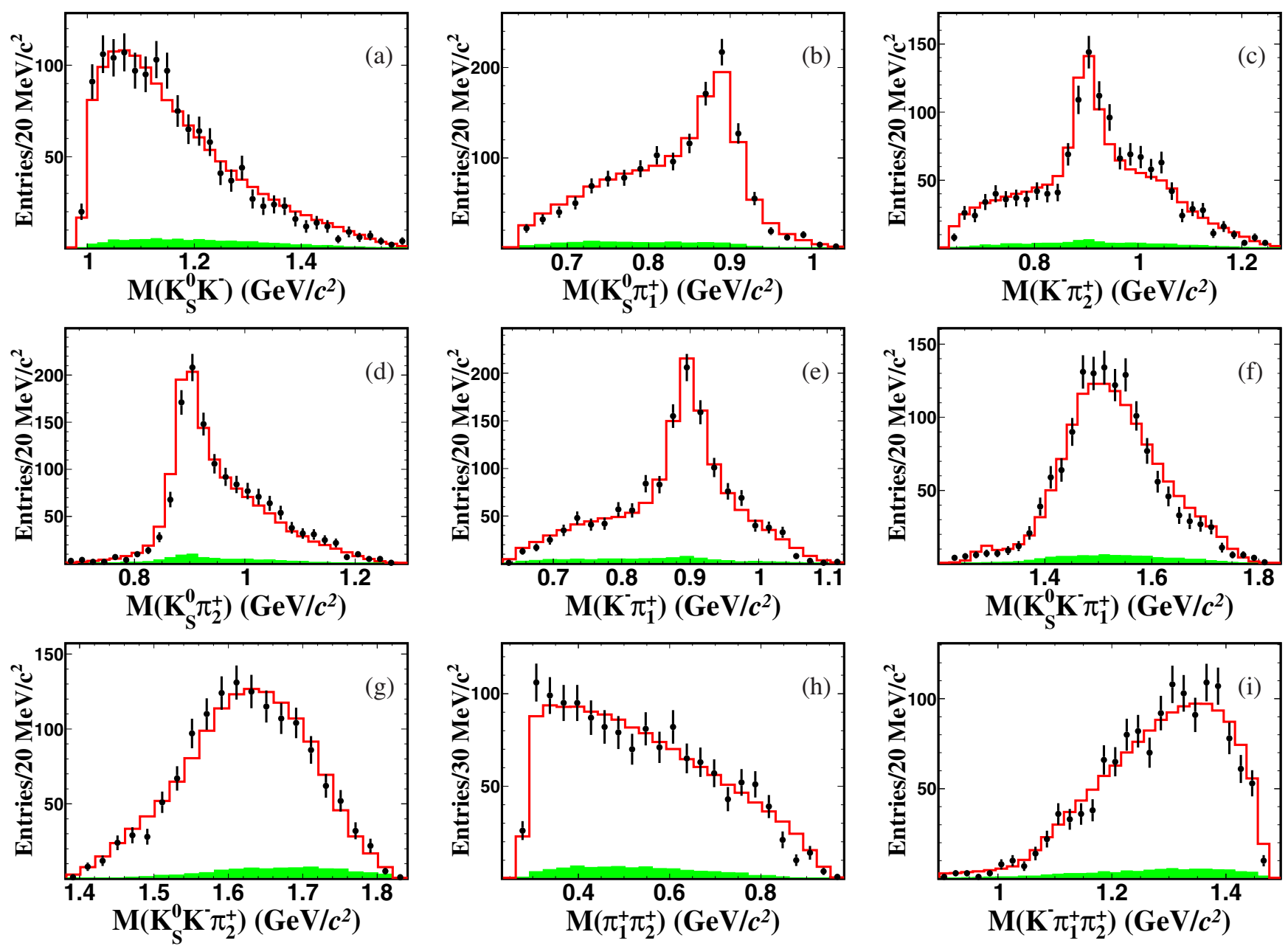

FIG. 2. The projections of (a) $M_{K_{S}^{0} K^{-}}$, (b) $M_{K_{S}^{0} \pi_{1}^{+}}$, (c) $M_{K^{-} \pi_{2}^{+}}$, (d) $M_{K_{S}^{0} \pi_{2}^{+}}$, (e) $M_{K^{-} \pi^{+}}$, (f) $M_{K_{\mathrm{S}}^{0} K^{-} \pi_{1}^{+}}$, (g) $M_{K_{\mathrm{S}}^{0} K^{-} \pi_{2}^{+}}$, (h) $M_{\pi_{1}^{-} \pi_{2}^{+}}$, and (i) $M_{K^{-} \pi_{1}^{+} \pi_{2}^{+}}$for the nominal amplitude fit are shown from data samples at $E_{\mathrm{cm}}$ between 4.178 and $4.226 \mathrm{GeV}$. The black points with error bars are data, the red histograms are the results of the nominal amplitude fit, and the green shaded histograms are the scaled GMC combinatorial background. For the identical pions, the one giving a lower $K_{S}^{0} \pi^{+}$invariant mass is denoted as $\pi_{1}^{+}$and the other is denoted as $\pi_{2}^{+}$.

\section{G. Fit results}

In the fit, the magnitude $(\rho)$ and phase $(\phi)$ of $D_{s}^{+} \rightarrow$ $K^{*}(892)^{+} \bar{K}^{*}(892)^{0}$ with angular momentum $L=0$ between $K^{*}(892)^{+}$and $\bar{K}^{*}(892)^{0}$ are fixed to 1 and 0 , respectively, and the magnitudes and phases of the other contributions are kept floating. The masses and widths of all resonances are fixed to the corresponding PDG averages [8]. We consider possible resonant contributions from $a_{0}(980), K^{*}(892), K^{*}(1410), K^{*}(1430)$, $K_{1}(1270), \quad K_{1}(1400), \quad \eta(1295), \quad \eta(1405), \quad \eta(1475)$, $f_{1}(1285), f_{1}(1420), f_{1}(1510)$, and $\phi(1680)$, as well as nonresonant contributions. The isospin symmetry requires the magnitude and phases of the processes $D_{s}^{+} \rightarrow$ $\eta(1475) \pi^{+}, \eta(1475) \rightarrow \bar{K}^{*}(892)^{0} K_{S}^{0}$ and $D_{s}^{+} \rightarrow \eta(1475) \pi^{+}$, $\eta(1475) \rightarrow K^{*}(892)^{+} K^{-}$to be the same [30]. Resonant or nonresonant contributions with a significance of larger than 4 standard deviations are retained in the model, where the significance is calculated from the change of the loglikelihood values and the corresponding degrees of freedom. Eleven amplitude contributions are retained in the nominal fit, including a nonresonant component of $K^{*}(892)^{+} K^{-}$with $L=1$ between $K^{*}(892)^{+}$and $K^{-}$. All the resonant and nonresonant contributions and their $\phi$, FFs and significances are listed in Table III. The magnitude and correlation matrix are provided in the Supplemental Material [31]. The projections for the nine invariant-mass distributions are shown in Fig. 2.

To validate the fit performance, 300 sets of SMC samples with the same size as the data samples are generated according to the nominal fit results in this analysis. Each sample is analyzed with the same method as for data. The pull value is given by $V_{\text {pull }}=\left(V_{\text {fit }}-V_{\text {input }}\right) / \sigma_{\text {fit }}$, where $V_{\text {input }}$ is the input value in the generator, $V_{\text {fit }}$ and $\sigma_{\text {fit }}$ are the output value and the corresponding statistical uncertainty, respectively. The resulting pull distributions are fitted with 
TABLE IV. Summary of systematic uncertainties on the $\phi$ and FFs from different sources, in units of the corresponding statistical uncertainties: (I) $K \pi S$-wave model, (II) line shape of $a_{0}(980)$, (III) effective barrier radius, (IV) masses and widths of the resonances considered, (V) background estimation, (VI) experimental effects, and (VII) neglected resonances.

\begin{tabular}{|c|c|c|c|c|c|c|c|c|c|}
\hline \multirow[b]{2}{*}{ Component } & & \multicolumn{7}{|c|}{ Source } & \multirow[b]{2}{*}{ Total } \\
\hline & & $\mathrm{I}$ & II & III & IV & $\mathrm{V}$ & VI & VII & \\
\hline$D_{s}^{+}[S] \rightarrow K^{*}(892)^{+} \bar{K}^{*}(892)^{0}$ & $\mathrm{FF}$ & 0.18 & 0.12 & 0.41 & 0.43 & 0.09 & 0.04 & 1.55 & 1.67 \\
\hline$D_{s}^{+}[P] \rightarrow K^{*}(892)^{+} \bar{K}^{*}(892)^{0}$ & $\begin{array}{c}\phi \\
\mathrm{FF}\end{array}$ & $\begin{array}{l}0.02 \\
0.05\end{array}$ & $\begin{array}{l}0.03 \\
0.00\end{array}$ & $\begin{array}{l}0.03 \\
0.06\end{array}$ & $\begin{array}{l}0.06 \\
0.10\end{array}$ & $\begin{array}{l}0.01 \\
0.00\end{array}$ & $\begin{array}{l}0.00 \\
0.00\end{array}$ & $\begin{array}{l}0.43 \\
0.02\end{array}$ & $\begin{array}{l}0.44 \\
0.13\end{array}$ \\
\hline$D_{s}^{+}[D] \rightarrow K^{*}(892)^{+} \bar{K}^{*}(892)^{0}$ & $\begin{array}{c}\phi \\
\mathrm{FF}\end{array}$ & $\begin{array}{l}0.02 \\
0.04\end{array}$ & $\begin{array}{l}0.04 \\
0.05\end{array}$ & $\begin{array}{l}0.00 \\
0.26\end{array}$ & $\begin{array}{l}0.03 \\
0.06\end{array}$ & $\begin{array}{l}0.01 \\
0.00\end{array}$ & $\begin{array}{l}0.03 \\
0.00\end{array}$ & $\begin{array}{l}0.28 \\
0.23\end{array}$ & $\begin{array}{l}0.29 \\
0.36\end{array}$ \\
\hline$D_{s}^{+} \rightarrow K^{*}(892)^{+} \bar{K}^{*}(892)^{0}$ & $\mathrm{FF}$ & 0.18 & 0.00 & 0.24 & 0.52 & 0.00 & 0.00 & 1.55 & 1.66 \\
\hline$D_{s}^{+} \rightarrow K^{*}(892)^{+}\left(K^{-} \pi^{+}\right)_{S-\text { wave }}$ & $\begin{array}{c}\phi \\
\mathrm{FF}\end{array}$ & $\begin{array}{l}0.36 \\
0.08\end{array}$ & $\begin{array}{l}0.13 \\
0.05\end{array}$ & $\begin{array}{l}0.04 \\
0.06\end{array}$ & $\begin{array}{l}0.20 \\
0.02\end{array}$ & $\begin{array}{l}0.01 \\
0.00\end{array}$ & $\begin{array}{l}0.09 \\
0.00\end{array}$ & $\begin{array}{l}0.37 \\
0.85\end{array}$ & $\begin{array}{l}0.58 \\
0.86\end{array}$ \\
\hline$D_{s}^{+} \rightarrow \bar{K}^{*}(892)^{0}\left(K_{S}^{0} \pi^{+}\right)_{S-\text { wave }}$ & $\begin{array}{c}\phi \\
\mathrm{FF}\end{array}$ & $\begin{array}{l}0.48 \\
0.08\end{array}$ & $\begin{array}{l}0.08 \\
0.02\end{array}$ & $\begin{array}{l}0.06 \\
0.04\end{array}$ & $\begin{array}{l}0.24 \\
0.17\end{array}$ & $\begin{array}{l}0.01 \\
0.01\end{array}$ & $\begin{array}{l}0.03 \\
0.00\end{array}$ & $\begin{array}{l}0.87 \\
0.79\end{array}$ & $\begin{array}{l}1.03 \\
0.81\end{array}$ \\
\hline$D_{s}^{+} \rightarrow \eta(1475) \pi^{+}, \eta(1475) \rightarrow a_{0}(980)^{-} \pi^{+}$ & $\begin{array}{c}\phi \\
\mathrm{FF}\end{array}$ & $\begin{array}{l}0.01 \\
0.05\end{array}$ & $\begin{array}{l}0.35 \\
1.96\end{array}$ & $\begin{array}{l}0.00 \\
0.12\end{array}$ & $\begin{array}{l}0.12 \\
0.22\end{array}$ & $\begin{array}{l}0.02 \\
0.05\end{array}$ & $\begin{array}{l}0.05 \\
0.02\end{array}$ & $\begin{array}{l}0.26 \\
0.08\end{array}$ & $\begin{array}{l}0.45 \\
1.98\end{array}$ \\
\hline$D_{s}^{+} \rightarrow \eta(1475) \pi^{+}, \eta(1475) \rightarrow \bar{K}^{*}(892)^{0} K_{S}^{0}$ & $\begin{array}{c}\phi \\
\mathrm{FF}\end{array}$ & $\begin{array}{l}0.01 \\
0.02\end{array}$ & $\begin{array}{l}0.13 \\
0.02\end{array}$ & $\begin{array}{l}0.09 \\
0.02\end{array}$ & $\begin{array}{l}0.70 \\
0.04\end{array}$ & $\begin{array}{l}0.00 \\
0.00\end{array}$ & $\begin{array}{l}0.03 \\
0.00\end{array}$ & $\begin{array}{l}0.12 \\
0.29\end{array}$ & $\begin{array}{l}0.73 \\
0.30\end{array}$ \\
\hline $\begin{array}{l}D_{s}^{+} \rightarrow \eta(1475) \pi^{+}, \eta(1475) \rightarrow K^{*}(892)^{+} K^{-} \\
D_{s}^{+} \rightarrow \eta(1475) \pi^{+}, \eta(1475) \rightarrow K^{*}(892) K\end{array}$ & $\begin{array}{c}\phi \\
\mathrm{FF} \\
\mathrm{FF}\end{array}$ & $\begin{array}{l}0.01 \\
0.02 \\
0.04\end{array}$ & $\begin{array}{l}0.13 \\
0.02 \\
0.05\end{array}$ & $\begin{array}{l}0.09 \\
0.02 \\
0.05\end{array}$ & $\begin{array}{l}0.70 \\
0.04 \\
0.09\end{array}$ & $\begin{array}{l}0.00 \\
0.00 \\
0.01\end{array}$ & $\begin{array}{l}0.03 \\
0.00 \\
0.00\end{array}$ & $\begin{array}{l}0.12 \\
0.31 \\
0.66\end{array}$ & $\begin{array}{l}0.73 \\
0.31 \\
0.68\end{array}$ \\
\hline$D_{s}^{+} \rightarrow \eta(1475) \pi^{+}, \eta(1475) \rightarrow\left(K_{S}^{0} \pi^{+}\right)_{S-\text { wave }} K^{-}$ & $\begin{array}{c}\phi \\
\mathrm{FF}\end{array}$ & $\begin{array}{l}0.48 \\
0.25\end{array}$ & $\begin{array}{l}0.08 \\
0.62\end{array}$ & $\begin{array}{l}0.01 \\
0.23\end{array}$ & $\begin{array}{l}0.40 \\
0.80\end{array}$ & $\begin{array}{l}0.01 \\
0.00\end{array}$ & $\begin{array}{l}0.07 \\
0.00\end{array}$ & $\begin{array}{l}0.26 \\
0.46\end{array}$ & $\begin{array}{l}0.68 \\
1.16\end{array}$ \\
\hline$D_{s}^{+} \rightarrow f_{1}(1285) \pi^{+}, f_{1}(1285) \rightarrow a_{0}(980)^{-} \pi^{+}$ & $\begin{array}{c}\phi \\
\mathrm{FF}\end{array}$ & $\begin{array}{l}0.03 \\
0.01\end{array}$ & $\begin{array}{l}0.05 \\
0.45\end{array}$ & $\begin{array}{l}0.02 \\
0.01\end{array}$ & $\begin{array}{l}0.18 \\
0.08\end{array}$ & $\begin{array}{l}0.00 \\
0.00\end{array}$ & $\begin{array}{l}0.02 \\
0.00\end{array}$ & $\begin{array}{l}0.48 \\
0.12\end{array}$ & $\begin{array}{l}0.52 \\
0.48\end{array}$ \\
\hline $\begin{array}{l}D_{S}^{+} \rightarrow\left(K^{*}(892)^{+} K^{-}\right)_{P} \pi^{+} \\
\left(K^{*}(892)^{+} K^{-}\right)_{P} \rightarrow K^{*}(892)^{+} K^{-}\end{array}$ & $\begin{array}{c}\phi \\
\mathrm{FF}\end{array}$ & $\begin{array}{l}0.01 \\
0.05\end{array}$ & $\begin{array}{l}0.03 \\
0.19\end{array}$ & $\begin{array}{l}0.06 \\
0.18\end{array}$ & $\begin{array}{l}0.09 \\
0.03\end{array}$ & $\begin{array}{l}0.00 \\
0.06\end{array}$ & $\begin{array}{l}0.02 \\
0.02\end{array}$ & $\begin{array}{l}0.21 \\
0.87\end{array}$ & $\begin{array}{l}0.24 \\
0.91\end{array}$ \\
\hline
\end{tabular}

Gaussian distributions. The fitted mean value of the pull distribution for the FF of $D_{s}^{+} \rightarrow \eta(1475) \pi^{+}, \eta(1475) \rightarrow$ $\left(K_{S}^{0} \pi^{+}\right)_{S-\text { wave }} K^{-}$deviates from zero by more than $3.0 \sigma$; we correct its FF according to the deviation and the uncertainty of the FF.

\section{H. Systematic uncertainties}

The systematic uncertainties for the amplitude analysis are studied in the following categories.

(i) $K \pi S$-wave model: The fixed parameters of the model are evaluated by varying the input values within $\pm 1 \sigma$ according to Ref. [28].

(ii) Line shape of $a_{0}(980)$ : The Flatté parameters are shifted by $\pm 1 \sigma$ based on the values given in Ref. [27].

(iii) Effective barrier radius: The barrier radius is varied within $\pm 1 \mathrm{GeV}^{-1} \times \hbar c$ for intermediate resonances and the $D_{s}^{+}$meson.

(iv) Masses and widths of the resonances considered: The masses and widths are shifted by $\pm 1 \sigma$ based on their values from the PDG [8]. (v) Background estimation: We shift the fractions of the signal in Eq. (9) according to the uncertainty associated with the background estimation and take the largest shift as the systematic uncertainty.

(vi) Experimental effects: To determine the systematic uncertainty due to tracking and PID efficiencies, we alter the fit by shifting the $\gamma_{\epsilon}$ in Eq. (6) within its uncertainty, and the change of the nominal fit result is taken as the systematic uncertainty.

(vii) Neglected resonances: The intermediate processes with statistical significance less than 4 standard deviations are added one-by-one to the nominal contributions. For each parameter, the maximum difference with respect to the nominal fit result is taken as the corresponding systematic uncertainty.

(viii) Fit uncertainties: The fitted widths from the pull distributions described in Sec. IV G are consistent with 1.0 within $2.0 \sigma$. Therefore, the fit uncertainties are estimated properly and no systematic uncertainty is assigned from this source.

All of the systematic uncertainties of the $\phi$ and FFs are listed in Table IV. The total systematic uncertainties are 
obtained by adding the above systematic uncertainties in quadrature.

\section{BRANCHING-FRACTION MEASUREMENT}

\section{A. Yields and efficiencies}

The selection criteria of the tagged $D_{s}^{-}$and signal $D_{s}^{+}$ candidates are the same as in Sec. III, except for the following requirements: (i) the requirement of the secondary vertex fit for $K_{S}^{0}$ from the tag modes is removed, while that for the signal is retained; (ii) a further requirement of $p_{\pi^{ \pm} / \pi^{0}}>$ $0.1 \mathrm{GeV} / c$ is added to remove the soft $\pi^{ \pm} / \pi^{0}$ directly from $D^{* \pm} / D^{* 0}$ decays; (iii) the tagged $D_{s}^{-}$candidates are reconstructed by looping over all their daughter tracks to form different combinations. If there are multiple candidates from the same event, the one with $M_{\text {rec }}$ closest to the $D_{s}^{* \pm}$ mass is accepted; (iv) at least one of the $D_{s}^{+} / D_{s}^{-}$candidates must satisfy $M_{\text {rec }}>2.10 \mathrm{GeV} / c^{2}$; (v) the combination with average mass $\bar{M}=\left[M_{\text {tag }}+M_{\text {sig }}\right] / 2$ closest to the nominal mass of $D_{s}^{+}$[8] is chosen among the multiple candidates.

The ST yields $\left(N_{\mathrm{ST}}\right)$ and DT yields $\left(N_{\mathrm{DT}}\right)$ in data are determined by fitting the $M_{\text {tag }}$ distributions from different tag modes and $M_{\text {sig }}$ distributions, respectively. In each fit, the signal shape is modeled using the simulated shape convolved with a Gaussian function, whose resolution and mean are free parameters, and the background is described with a secondorder Chebychev polynomial. These fits give a total ST yield of $N_{\mathrm{ST}}=550496 \pm 2411$. The $M_{\mathrm{tag}}$ distributions at $E_{\mathrm{cm}}=$ $4.178 \mathrm{GeV}$ are shown in Fig. 3 as an example. The total DT signal yield, $N_{\mathrm{DT}}^{\text {tot }}$, is determined to be $1332 \pm 42$, as shown in Fig. 4. The fits to the $M_{\text {sig }}$ distribution for GMC are performed to estimate the corresponding ST efficiencies $\left(\epsilon_{\mathrm{ST}}\right)$. The DT efficiencies $\left(\epsilon_{\mathrm{DT}}\right)$ are determined by GMC, in which our amplitude analysis model is taken for the generation of the signal mode.

\section{B. Tagging technique and branching fraction}

The branching fraction for the signal mode is given by

$$
\mathcal{B}_{\mathrm{sig}}=\frac{N_{\mathrm{DT}}^{\mathrm{tot}}}{\sum_{i} \sum_{j} N_{\mathrm{ST}}^{i j} \cdot \epsilon_{\mathrm{DT}}^{i j} / \epsilon_{\mathrm{ST}}^{i j}},
$$

where the indices $i$ and $j$ denote the $i$ th tag mode and the $j$ th center-of-mass energy point. The $N_{\mathrm{ST}}^{i j}$ and $\epsilon_{\mathrm{ST}(\mathrm{DT})}^{i j}$ are
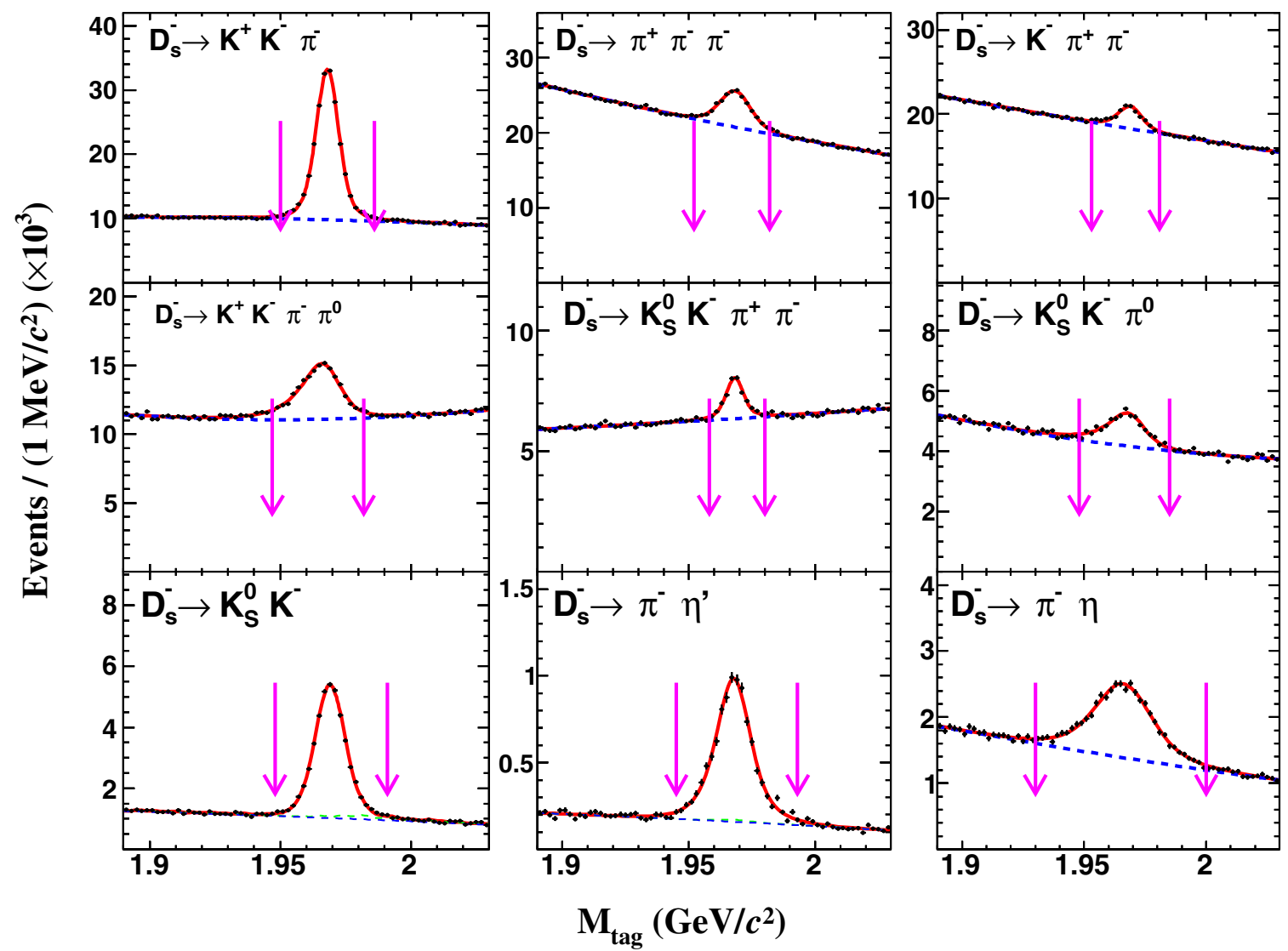

FIG. 3. Best-fit results to the $M_{\text {tag }}$ distributions of the ST candidates from the data sample taken at $E_{\mathrm{cm}}=4.178 \mathrm{GeV}$. The points with error bars are data. The red solid curves are the fit results. The blue dotted curves are the fitted background shapes. The pair of pink arrows indicates the chosen signal regions. The green dotted curve in the $D_{s}^{-} \rightarrow K_{S}^{0} K^{-}\left(D_{s}^{-} \rightarrow \pi^{-} \eta^{\prime}\right)$ mode is the $D_{s}^{-} \rightarrow K_{S}^{0} \pi^{-}$ $\left(D_{s}^{-} \rightarrow \pi^{+} \pi^{-} \pi^{-} \eta\right)$ component. 


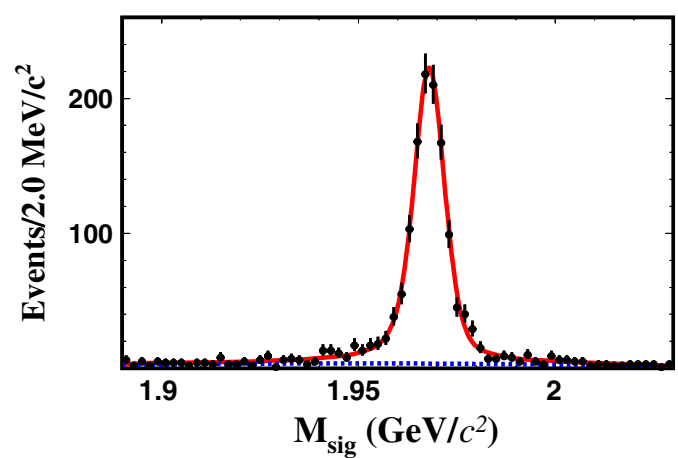

FIG. 4. Best-fit result to the $M_{\text {sig }}$ distributions of the DT candidates from data samples at $E_{\mathrm{cm}}$ between 4.178 and $4.226 \mathrm{GeV}$. The points with error bars are data. The red solid curve is the fit result. The blue dotted curve is the fitted background shape.

the number of the ST candidates and the corresponding ST (DT) detection efficiency.

Using Eq. (21) and the PDG value of the $\mathcal{B}\left(K_{S}^{0} \rightarrow\right.$ $\left.\pi^{+} \pi^{-}\right)=(69.20 \pm 0.05) \%$ [8], the absolute branching fraction can be obtained,

$$
\mathcal{B}\left(D_{s}^{+} \rightarrow K_{S}^{0} K^{-} \pi^{+} \pi^{+}\right)=(1.46 \pm 0.05) \%,
$$

where the uncertainty is statistical.

\section{SYSTEMATIC UNCERTAINTIES}

The systematic uncertainties for the branching-fraction measurement are studied in the following categories:

(i) $K^{ \pm}$and $\pi^{ \pm}$tracking (PID) efficiencies: The tracking (PID) efficiencies are studied using samples of $e^{+} e^{-} \rightarrow K^{+} K^{-} \pi^{+} \pi^{-} \quad\left[e^{+} e^{-} \rightarrow K^{+} K^{-} K^{+} K^{-}\right.$, $K^{+} K^{-} \pi^{+} \pi^{-}\left(\pi^{0}\right)$ and $\left.\pi^{+} \pi^{-} \pi^{+} \pi^{-}\left(\pi^{0}\right)\right]$ events. The systematic uncertainties for $K^{ \pm}$and $\pi^{ \pm}$due to tracking (PID) are estimated to be $0.8 \%$ and $0.3 \%$ $(0.8 \%$ and $0.5 \%)$, respectively.

(ii) $K_{S}^{0}$ reconstruction efficiency: The uncertainty for the $K_{S}^{0}$ reconstruction efficiency is assigned as $1.5 \%$ per $K_{S}^{0}$, obtained using control samples of $J / \psi \rightarrow$ $K_{S}^{0} K^{ \pm} \pi^{\mp}$ and $\phi K_{S}^{0} K^{ \pm} \pi^{\mp}$ decays.

(iii) Fit to the DT $M_{\text {sig }}$ distribution: The uncertainty associated with the modeling of the DT $M_{\text {sig }}$ distribution is studied with alternative models for signal and background. The uncertainties are estimated by comparing with the fit results obtained using the signal and background shapes directly from the simulated samples.

(iv) Fit to the ST $M_{\text {tag }}$ distribution: We change the background shape from the second-order Chebychev polynomial to a third-order Chebychev polynomial, causing a $0.18 \%$ relative change of the branching fraction. The systematic uncertainty due
TABLE V. Systematic uncertainties in the branching-fraction measurement.

\begin{tabular}{lc}
\hline \hline Source & Uncertainty $(\%)$ \\
\hline Tracking efficiency & 1.4 \\
PID efficiency & 1.8 \\
$K_{S}^{0}$ reconstruction efficiency & 1.5 \\
DT $M_{\text {sig fit }}$ & 1.7 \\
ST $M_{\text {tag fit }}$ & 0.2 \\
Measurement method & 0.3 \\
Statistics of simulated events & 0.3 \\
Amplitude analysis model & 0.6 \\
$\mathcal{B}\left(K_{S}^{0} \rightarrow \pi^{+} \pi^{-}\right)[8]$ & 0.1 \\
Total & 3.3 \\
\hline \hline
\end{tabular}

to the modeling of the signal distribution is determined to be $0.16 \%$ by performing an alternative fit using the shape directly obtained from the simulated sample. The quadratic sum of these terms, $0.24 \%$, is assigned as the systematic uncertainty.

(v) Measurement method: The possible bias due to the measurement method is estimated to be $0.3 \%$ by comparing the measured branching fraction in the SMC, using the same method as in data analysis, to the value input in the SMC generation.

(vi) Statistics of simulated events: The uncertainty associated with the limited statistics of GMC for the detection efficiency is $0.3 \%$.

(vii) Amplitude analysis model: The uncertainty from the amplitude analysis model is $0.6 \%$, estimated from the efficiency difference obtained by varying the fitted parameters $c_{n}$ in Eq. (3) according to the error matrix.

All the systematic uncertainties of the branching-fraction measurement are listed in Table V. When added in quadrature, they sum to a relative uncertainty of $3.3 \%$, which is the same as the statistical uncertainty on the measurement.

\section{CONCLUSION}

Using $6.32 \mathrm{fb}^{-1}$ of $e^{+} e^{-}$collision data collected by the BESIII detector with center-of-mass energies between 4.178 and $4.226 \mathrm{GeV}$, we report the first amplitude analysis of the $D_{s}^{+} \rightarrow K_{S}^{0} K^{-} \pi^{+} \pi^{+}$decays and an improved measurement of the $D_{s}^{+} \rightarrow K_{S}^{0} K^{-} \pi^{+} \pi^{+}$branching fraction. The model indicates that the quasi-two-body decay $D_{s}^{+} \rightarrow$ $K^{*}(892)^{+} \bar{K}^{*}(892)^{0}$ is dominant, with a fit fraction of $\left(40.6 \pm 2.9_{\text {stat }} \pm 4.9_{\text {sys }}\right) \%$. In addition, there are significant contributions from $f_{1}(1285), \eta(1475)$, and $\left(K^{*}(892)^{+} K^{-}\right)_{P}$ in the mass spectrum of $K_{S}^{0} K^{-} \pi^{+}$. The $\eta(1475)$ meson decays to both $K^{*} K$ and $a_{0}(980) \pi$ final states, while the $f_{1}(1285)$ meson decays only to $a_{0}(980) \pi$. The absolute branching fraction of the $D_{s}^{+} \rightarrow K_{S}^{0} K^{-} \pi^{+} \pi^{+}$decay is determined to be $\left(1.46 \pm 0.05_{\text {stat }} \pm 0.05_{\text {sys }}\right) \%$, and the branching fractions for different components are listed in Table VI. 
TABLE VI. The branching fractions measured in this analysis and from PDG [8]. The $K^{*}(892)^{+}, \bar{K}^{*}(892)^{0}$, and $a_{0}(980)^{-}$denote $K^{*}(892)^{+} \rightarrow K_{S}^{0} \pi^{+}, \bar{K}^{*}(892)^{0} \rightarrow K^{-} \pi^{+}$, and $a_{0}(980)^{-} \rightarrow K_{S}^{0} K^{-}$, respectively.

\begin{tabular}{lcc}
\hline \hline & \multicolumn{1}{c}{$\mathrm{BF}\left(10^{-3}\right)$} \\
Process & This analysis & PDG \\
\hline$D_{s}^{+}[S] \rightarrow K^{*}(892)^{+} \bar{K}^{*}(892)^{0}$ & $5.01 \pm 0.49 \pm 0.78$ & \\
$D_{s}^{+}[P] \rightarrow K^{*}(892)^{+} \bar{K}^{*}(892)^{0}$ & $1.10 \pm 0.16 \pm 0.10$ & \\
$D_{s}^{+}[D] \rightarrow K^{*}(892)^{+} \bar{K}^{*}(892)^{0}$ & $0.65 \pm 0.12 \pm 0.10$ & $7.98 \pm 2.88$ \\
$D_{s}^{+} \rightarrow K^{*}(892)^{+} \bar{K}^{*}(892)^{0}$ & $5.93 \pm 0.47 \pm 0.74$ & \\
$D_{s}^{+} \rightarrow K^{*}(892)^{+}\left(K^{-} \pi^{+}\right)_{S-\text { wave }}$ & $0.73 \pm 0.17 \pm 0.15$ & \\
$D_{s}^{+} \rightarrow \bar{K}^{*}(892)^{0}\left(K_{S}^{0} \pi^{+}\right)_{S-\text { wave }}$ & $1.06 \pm 0.16 \pm 0.13$ & \\
$D_{s}^{+} \rightarrow \eta(1475) \pi^{+}, \eta(1475) \rightarrow a_{0}(980)^{-} \pi^{+}$ & $1.57 \pm 0.39 \pm 0.76$ & \\
$D_{s}^{+} \rightarrow \eta(1475) \pi^{+}, \eta(1475) \rightarrow \bar{K}^{*}(892)^{0} K_{S}^{0}$ & $0.32 \pm 0.10 \pm 0.10$ & \\
$D_{s}^{+} \rightarrow \eta(1475) \pi^{+}, \eta(1475) \rightarrow K^{*}(892)^{+} K^{-}$ & $0.32 \pm 0.10 \pm 0.10$ & \\
$D_{s}^{+} \rightarrow \eta(1475) \pi^{+}, \eta(1475) \rightarrow K^{*}(892) K^{+}$ & $0.72 \pm 0.21 \pm 0.14$ & \\
$D_{s}^{+} \rightarrow \eta(1475) \pi^{+}, \eta(1475) \rightarrow\left(K_{S}^{0} \pi^{+}\right)_{S-\text { wave }} K^{-}$ & $3.44 \pm 0.54 \pm 1.10$ & \\
$D_{s}^{+} \rightarrow f_{1}(1285) \pi^{+}, f_{1}(1285) \rightarrow a_{0}(980)^{-} \pi^{+}$ & $0.33 \pm 0.08 \pm 0.10$ & \\
$D_{s}^{+} \rightarrow\left(K^{*}(892)^{+} K^{-}\right)_{P} \pi^{+},\left(K^{*}(892)^{+} K^{-}\right)_{P} \rightarrow K^{*}(892)^{+} K^{-}$ & $1.58 \pm 0.28 \pm 0.26$ & \\
$D_{s}^{+} \rightarrow K_{S}^{0} K^{-} \pi^{+} \pi^{+}$ & $14.60 \pm 0.46 \pm 0.48$ & \\
\hline \hline
\end{tabular}

The branching fraction of the quasi-two-body decay $D_{s}^{+} \rightarrow$ $K^{*}(892)^{+} \bar{K}^{*}(892)^{0}$ is calculated to be $\left(5.34 \pm 0.39_{\text {stat }} \pm\right.$ $\left.0.64_{\text {sys }}\right) \%$. Our measurements are consistent with the current world averages [8] but much more precise.

\section{ACKNOWLEDGMENTS}

The BESIII Collaboration thanks the staff of BEPCII and the IHEP computing center for their strong support. This work was supported in part by National Key Research and Development Program of China under Contracts No. 2020YFA0406400 and No. 2020YFA0406300; National Natural Science Foundation of China (NSFC) under Contracts No. 11625523, No. 11635010, No. 11735014, No. 11822506, No. 11835012, No. 11935015, No. 11935016, No. 11935018, and No. 11961141012; the Chinese Academy of Sciences (CAS) Large-Scale Scientific Facility Program; Joint Large-Scale Scientific Facility Funds of the NSFC and CAS under Contracts No. U1732263, No. U1832107, No. U1832207, and No. U2032104; CAS Key Research
Program of Frontier Sciences under Contracts No. QYZDJSSW-SLH003 and No. QYZDJ-SSW-SLH040; 100 Talents Program of CAS; Institute of Nuclear and Particle Physics (INPAC) and Shanghai Key Laboratory for Particle Physics and Cosmology; ERC under Contract No. 758462; European Union Horizon 2020 research and innovation programme under Contract No. Marie Sklodowska-Curie Grant No. 894790; German Research Foundation DFG under Contracts No. 443159800, Collaborative Research Center CRC 1044, FOR 2359, FOR 2359, GRK 214; Istituto Nazionale di Fisica Nucleare, Italy; Ministry of Development of Turkey under Contract No. DPT2006K120470; National Science and Technology fund; Olle Engkvist Foundation under Contract No. 200-0605; STFC (United Kingdom); the Knut and Alice Wallenberg Foundation (Sweden) under Contract No. 2016.0157; The Royal Society, UK under Contracts No. DH140054 and No. DH160214; the Swedish Research Council; U.S. Department of Energy under Contracts No. DE-FG02-05ER41374 and No. DE-SC-0012069.
[1] M. Ablikim et al. (BESIII Collaboration), Phys. Rev. D 99, 112005 (2019).

[2] J. P. Alexander et al. (CLEO Collaboration), Phys. Rev. D 79, 052001 (2009).

[3] M. Ablikim et al. (BESIII Collaboration), Phys. Rev. Lett. 122, 071802 (2019).

[4] M. Ablikim et al. (BESIII Collaboration), Phys. Rev. Lett. 122, 061801 (2019).
[5] M. Ablikim et al. (BESIII Collaboration), Phys. Rev. Lett. 122, 121801 (2019).

[6] P. U. E. Onyisi et al. (CLEO Collaboration), Phys. Rev. D 88, 032009 (2013).

[7] H. Albrecht et al. (ARGUS Collaboration), Z. Phys. C 53, 361 (1992).

[8] P. A. Zyla et al. (Particle Data Group), Prog. Theor. Exp. Phys. 2020, 083C01 (2020). 
[9] X. W. Kang and H. B. Li, Phys. Lett. B 684, 137 (2010).

[10] M. Ablikim et al. (BESIII Collaboration), Phys. Rev. D 97, 051101 (2018).

[11] M. Ablikim et al. (BESIII Collaboration), Phys. Rev. D 97, 072014 (2018).

[12] J. Abdallah et al. (DELPHI Collaboration), Phys. Lett. B 569, 129 (2003).

[13] F. Nichitiu et al. (OBELXI Collaboration), Phys. Lett. B 545, 261 (2002).

[14] M. Ablikim et al. (BESIII Collaboration), Nucl. Instrum. Methods Phys. Res., Sect. A 614, 345 (2010).

[15] C. H. Yu et al., in Proceedings of IPAC2016, Busan, Korea, 2016, http://dx.doi.org/10.18429/JACoW-IPAC2016-TUYA01.

[16] X. Wang et al., J. Instrum. 11, C08009 (2016).

[17] S. Agostinelli et al. (GEANT4 Collaboration), Nucl. Instrum. Methods Phys. Res., Sect. A 506, 250 (2003).

[18] R. G. Ping, Chin. Phys. C 38, 083001 (2014).

[19] S. Jadach, B. F. L. Ward, and Z. Was, Phys. Rev. D 63, 113009 (2001); Comput. Phys. Commun. 130, 260 (2000).

[20] D. J. Lange, Nucl. Instrum. Methods Phys. Res., Sect. A 462, 152 (2001); R. G. Ping, Chin. Phys. C 32, 243 (2008).
[21] J. C. Chen, G. S. Huang, X. R. Qi, D. H. Zhang, and Y. S. Zhu, Phys. Rev. D 62, 034003 (2000); R. L. Yang, R. G. Ping, and H. Chen, Chin. Phys. Lett. 31, 061301 (2014).

[22] E. Richter-Was, Phys. Lett. B 303, 163 (1993).

[23] M. Xu et al., Chin. Phys. C 33, 1381 (2009).

[24] B. S. Zou and D. V. Bugg, Eur. Phys. J. A 16, 537 (2003).

[25] W. Verkerke and D. P. Kirkby, RooFit Users Manual v2.91.

[26] M. Ablikim et al. (BESIII Collaboration), Phys. Rev. D 95, 072010 (2017).

[27] M. Ablikim et al. (BESIII Collaboration), Phys. Rev. D 95, 032002 (2017).

[28] I. Adachi et al. (BABAR and Belle Collaborations), Phys. Rev. D 98, 112012 (2018).

[29] D. Aston et al., Nucl. Phys. B296, 493 (1988).

[30] J. J. Wu, X. H. Liu, Q. Zhao, and B. S. Zou, Phys. Rev. Lett. 108, 081803 (2012).

[31] See Supplemental Material at http://link.aps.org/supplemental/ 10.1103/PhysRevD.103.092006 for additional details on the magnitude and correlation matrix. 